\title{
PSRs J0248+6021 and J2240+5832: young pulsars in the northern Galactic plane
}

\section{Discovery, timing, and gamma-ray observations}

\author{
G. Theureau ${ }^{1}$, D. Parent ${ }^{2,3,4}$, I. Cognard ${ }^{1}$, G. Desvignes ${ }^{1,5}$, D. A. Smith ${ }^{4}$, J. M. Casandjian ${ }^{6}$, C. C. Cheung ${ }^{2,7}$, \\ H. A. Craig ${ }^{8}$, D. Donato ${ }^{9,10}$, R. Foster ${ }^{11}$, L. Guillemot ${ }^{4,12}$, A. K. Harding ${ }^{13}$, J.-F. Lestrade ${ }^{14}$, P. S. Ray ${ }^{2}$, \\ R. W. Romani ${ }^{8}$, D. J. Thompson ${ }^{13}$, W. W. Tian ${ }^{15,16}$, and K. Watters ${ }^{8}$
}

(Affiliations can be found after the references)

Received 1 July 2010 / Accepted 7 October 2010

\begin{abstract}
Context. Pulsars PSR J0248+6021 (with a rotation period $P=217 \mathrm{~ms}$ and spin-down power $\dot{E}=2.13 \times 10^{35} \mathrm{erg} \mathrm{s}^{-1}$ ) and PSR J2240+5832 $\left(P=140 \mathrm{~ms}, \dot{E}=2.12 \times 10^{35} \mathrm{erg} \mathrm{s}^{-1}\right)$ were discovered in 1997 with the Nançay radio telescope during a northern Galactic plane survey, using the Navy-Berkeley Pulsar Processor (NBPP) filter bank. The GeV gamma-ray pulsations from both were discovered using the Fermi Large Area Telescope.

Aims. We characterize the neutron star emission using radio and gamma-ray observations, and explore the rich environment of PSR J0248+6021. Methods. Twelve years of radio timing data, including glitches, with steadily improved instrumentation, such as the Berkeley-Orleans-Nançay pulsar backend, and a gamma-ray data set 2.6 times larger than previously published allow detailed investigations of these pulsars. Radio polarization data allow comparison with the geometry inferred from gamma-ray emission models.

Results. The two pulsars resemble each other in both radio and gamma-ray data. Both are rare in having a single gamma-ray pulse offset far from the radio peak. The anomalously high dispersion measure for PSR J0248+6021 $\left(D M=370 \mathrm{pc} \mathrm{cm}^{-3}\right)$ is most likely due to its being within the dense, giant HII region W5 in the Perseus arm at a distance of $2 \mathrm{kpc}$, as opposed to being beyond the edge of the Galaxy as obtained from models of average electron distributions. Its large transverse velocity and the low magnetic field along the line-of-sight favor this small distance. Neither gamma-ray, X-ray, nor optical data yield evidence of a pulsar wind nebula surrounding PSR J0248+6021. We report the discovery of gamma-ray pulsations from PSR J2240+5832. We argue that it could be in the outer arm, although slightly nearer than its $D M$-derived distance, but that it may be in the Perseus arm at half the distance.

Conclusions. The energy flux and distance yield a gamma-ray luminosity for PSR J0248+6021 of $L_{\gamma}=(1.4 \pm 0.3) \times 10^{34} \mathrm{erg} \mathrm{s}^{-1}$. For PSR J2240+5832, we find either $L_{\gamma}=(7.9 \pm 5.2) \times 10^{34} \mathrm{erg} \mathrm{s}^{-1}$ if the pulsar is in the outer arm, or $L_{\gamma}=(2.2 \pm 1.7) \times 10^{34} \mathrm{erg} \mathrm{s}^{-1}$ for the Perseus arm. These luminosities are consistent with an $L_{\gamma} \propto \sqrt{\dot{E}}$ rule. Comparison of the gamma-ray pulse profiles with model predictions, including the constraints obtained from radio polarization data, implies outer magnetosphere emission. These two pulsars differ mainly in terms of their inclination angles and acceleration gap widths, which in turn explain the observed differences in the gamma-ray peak widths.
\end{abstract}

Key words. gamma rays: stars - pulsars: individual: J0248+6021 - pulsars: individual: J2240+5832

\section{Introduction}

Pulsars PSR J0248+6021 and PSR J2240+5832 were discovered with the Nançay radio telescope (NRT) in a survey of the northern Galactic plane from 1997 to 1999 (Foster et al. 1997). Ray et al. (1999) reported their locations and rotation periods. Twelve years of timing measurements allow us to provide accurate ephemerides, including a proper motion determination for PSR J0248+6021 that helps us understand its relation to the complex of gas clouds co-located on the sky. Polarization data provides geometry constraints in addition to those obtained by comparing the gamma-ray pulse profiles with model predictions.

By coincidence, both pulsars have a high spin-down power $\dot{E}=2.1 \times 10^{35} \mathrm{erg} \mathrm{s}^{-1}$, making it also likely that they emit in GeV gamma-rays, as discussed by Smith et al. (2008). Indeed, PSR J0248+6021 is among the 46 gamma-ray pulsars described in the "First Pulsar Catalog" (Abdo et al. 2010b) using the Large Area Telescope (LAT) on the Fermi Gamma-ray Space Telescope (formerly GLAST). They have the same magnetic field strengths at the neutron-star light cylinder, $B_{\mathrm{LC}}$, to within $20 \%$, and the characteristic ages $\tau_{\mathrm{c}}=P / 2 \dot{P}$ differ by only a factor of two. Table 1 lists measured and derived parameters for both pulsars. PSR J2240+5832 has a smaller dispersion measure $\left(D M=263.5 \mathrm{pc} \mathrm{cm}^{-3}\right)$ than PSR J0248+6021 $(D M=$ $370 \mathrm{pc} \mathrm{cm}^{-3}$ ), and the NE2001 model of the Galactic electron distribution (Cordes \& Lazio 2002) assigns PSR J2240+5832 a distance of $10.3_{-3.3}^{+\infty} \mathrm{kpc}$, whereas it places PSR J0248+6021 well beyond the edge of the Galaxy ( $>43.5 \mathrm{kpc}$ ). Curiously however, it is the nominally closer pulsar, PSR J2240+5832 that pulsates less brightly in the Fermi data.

Two obvious solutions to this seeming paradox are that either there is some intrinsic difference between the pulsars, such as their orientation angles and beaming, or that the distances are simply misunderstood. In this paper, we clarify the situation.

\section{Nançay northern Galactic plane survey}

The NRT is a meridian instrument, with a primary antenna $200 \mathrm{~m}$ wide by $35 \mathrm{~m}$ tall, equivalent to a parabolic dish with a $94 \mathrm{~m}$ diameter. It can track objects with declinations $\delta>-39^{\circ}$ for 
Table 1. Measured and derived parameters for pulsars PSR J0248+6021 and PSR J2240+5832.

\begin{tabular}{|c|c|c|}
\hline Pulsar name........... & J0248+6021 & $\mathrm{J} 2240+5832$ \\
\hline Right ascension, (J2000) & $02: 48: 18.617(1)$ & $22: 40: 42.939(4)$ \\
\hline Declination, $(\mathrm{J} 2000) \ldots$. & $+60: 21: 34.72(1)$ & $+58: 32: 37.52(4)$ \\
\hline Position epoch, (MJD) .......... & 54000 & 54000 \\
\hline Galactic longitude, $l$ (degrees) & 136.9 & 106.6 \\
\hline Galactic latitude, $b$ (degrees).. & 0.698 & -0.111 \\
\hline Pulse frequency, $v\left(\mathrm{~s}^{-1}\right) \ldots \ldots \ldots \ldots \ldots \ldots$ & 4.6063 & 7.1462 \\
\hline First derivative of pulse frequency, $\dot{v}\left(10^{-12} \mathrm{~s}^{-2}\right) \ldots$ & -1.169 & -0.7843 \\
\hline 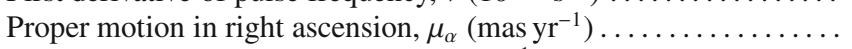 & $48 \pm 10$ & $-6.1 \pm 0.8$ \\
\hline Proper motion in declination, $\mu_{\delta}\left(\operatorname{mas}^{-1}\right) \ldots$ & $48 \pm 4$ & $-21.0 \pm 0.4$ \\
\hline Timing data span (MJD)............... & 54682.7 to 55160.9 & 54682.7 to 55202.6 \\
\hline Rms timing residual $(\mu s) \ldots \ldots \ldots \ldots \ldots$ & 181 & 66 \\
\hline Radio pulse width at $1.4 \mathrm{GHz}, W_{50}$ (phase) & 0.12 & 0.07 \\
\hline Dispersion measure, $D M\left(\mathrm{~cm}^{-3} \mathrm{pc}\right) \ldots \ldots \ldots$ & $370 \pm 1$ & $263.50 \pm 0.05$ \\
\hline$D M$ epoch, (MJD) $\ldots \ldots \ldots \ldots \ldots \ldots$ & 54000 & 54000 \\
\hline Rotation measure, $R M\left(\mathrm{rad} \mathrm{m}^{-2}\right) \ldots \ldots \ldots \ldots \ldots \ldots \ldots \ldots \ldots \ldots$ & $-158 \pm 6$ & $24 \pm 4$ \\
\hline Mean flux density at $1400 \mathrm{MHz}, S_{1400}(\mathrm{mJy}) \ldots \ldots \ldots \ldots \ldots \ldots$ & $13.7 \pm 2.7$ & $2.7 \pm 0.7$ \\
\hline 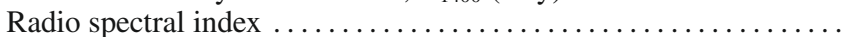 & $-1.2 \pm 0.1$ & $-1.8 \pm 0.3$ \\
\hline Radio-gamma-ray peak offset, $\delta \ldots \ldots \ldots \ldots \ldots \ldots \ldots \ldots$ & $0.39 \pm 0.02$ & $0.58 \pm 0.01$ \\
\hline \multicolumn{3}{|c|}{ Gamma-ray spectral parameters } \\
\hline Differential photon flux normalization, $N_{0}\left(10^{-11} \mathrm{~cm}^{-2} \mathrm{~s}^{-1} \mathrm{MeV}^{-1}\right)$ & $1.5 \pm 0.7 \pm 0.1$ & $0.13 \pm 0.06 \pm 0.10$ \\
\hline Integral photon flux, $F_{100}\left(>100 \mathrm{MeV}, 10^{-8} \mathrm{ph} \mathrm{cm}^{-2} \mathrm{~s}^{-1}\right) \ldots \ldots \ldots$ & $4.0 \pm 0.4 \pm 0.5$ & $1.5 \pm 0.8 \pm 0.4$ \\
\hline Integral energy flux, $G_{100}\left(>100 \mathrm{MeV}, 10^{-11} \mathrm{erg} \mathrm{cm}^{-2} \mathrm{~s}^{-1}\right) \ldots \ldots$ & $2.9 \pm 0.3 \pm 0.2$ & $1.0 \pm 0.4 \pm 0.2$ \\
\hline Exponential cut-off energy, $E_{\mathrm{c}}(\mathrm{GeV}) \ldots \ldots \ldots \ldots \ldots \ldots \ldots \ldots$ & $1.2 \pm 0.2 \pm 0.1$ & $5.7 \pm 4.4 \pm 1.0$ \\
\hline 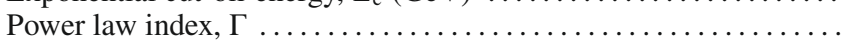 & $1.3 \pm 0.1 \pm 0.1$ & $1.8 \pm 0.6 \pm 0.1$ \\
\hline \multicolumn{3}{|l|}{ Derived quantities } \\
\hline Spin-down power, $\dot{E}\left(\mathrm{erg} \mathrm{s}^{-1}\right)$ & $2.13 \times 10^{35}$ & $2.12 \times 10^{35}$ \\
\hline 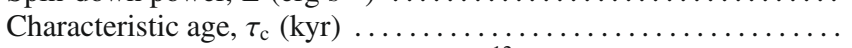 & 63 & 151 \\
\hline Surface magnetic field strength, $B_{\mathrm{S}}\left(10^{12} \mathrm{G}\right) \ldots \ldots \ldots \ldots \ldots \ldots \ldots$ & 3.5 & 1.45 \\
\hline Magnetic field strength at the light cylinder, $B_{\mathrm{LC}}(\mathrm{G}) \ldots \ldots \ldots \ldots$ & 3150 & 4860 \\
\hline Distance from NE2001, $d_{\mathrm{NE}}(\mathrm{kpc}) \ldots \ldots \ldots \ldots \ldots \ldots \ldots \ldots \ldots$ & $>43.5$ & $10.3_{-3.3}^{+\infty}$ \\
\hline 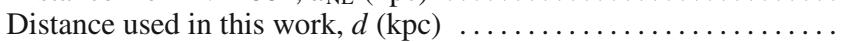 & $2.0 \pm 0.2$ & $7.7 \pm 0.7$ or $3.8 \pm 0.8$ \\
\hline Luminosity, $L_{\gamma}\left(E>100 \mathrm{MeV}, 10^{34} \mathrm{erg} \mathrm{s}^{-1}\right) \ldots \ldots \ldots \ldots \ldots \ldots$ & $1.4 \pm 0.3$ & $7.9 \pm 5.2$ or $2.2 \pm 1.7$ \\
\hline Efficiency, $\eta=L_{\gamma} / \dot{E}(\%) \ldots \ldots \ldots \ldots \ldots$ & $7 \pm 2$ & $37 \pm 25$ or $10 \pm 8$ \\
\hline
\end{tabular}

roughly one hour around culmination. The half-power beam width at $1.4 \mathrm{GHz}$ is 4 arcmin (east-west) by 22 arcmin (northsouth) at $\delta=0^{\circ}$, a shape well-adapted to sky scanning. The $1.4 \mathrm{GHz}$ nominal system temperature at the time of these observations was about $50 \mathrm{~K}$ in both horizontal and vertical polarizations, for $\delta \simeq 60^{\circ}$. The "FORT" receiver upgrade from 1999 to 2001 improved sensitivity by a factor of 2.2 , with an efficiency of $1.4 \mathrm{~K} / \mathrm{Jy}$ and a system temperature of $35 \mathrm{~K}$ at $1.4 \mathrm{GHz}$. The frequency coverage is now continuous from $1.1 \mathrm{GHz}$ to $3.5 \mathrm{GHz}$ and allows full Stokes measurements (Theureau et al. 2005).

Two pulsar instrumentation systems ("backends") were used successively, first for the survey and then for the continued timing. The earlier system was the Navy-Berkeley Pulsar Processor (NBPP) filter bank, designed and built for this survey at the Naval Research Laboratory (NRL) in collaboration with the University of California, Berkeley. We set the 96 channels in the full crate to cover $1.5 \mathrm{MHz} / \mathrm{channel}$, for a total bandwidth of $144 \mathrm{MHz}$ (Backer et al. 1997). The output data has a time resolution of 50 to $100 \mu \mathrm{s}$ in search mode, allowing detection of signals with periods as short as $0.1 \mathrm{~ms}$. For the survey, data were acquired at a sampling rate of $60 \mu$ s using 4-bit digitization of 96 channels and both polarizations.

Since 2004, the NRT pulsar backend is the BON (BerkeleyOrléans-Nançay) coherent dedispersor with a bandwidth of $128 \mathrm{MHz}$. A spectrometer digitizes data voltages, followed by four data servers that share the data over a 70-node cluster of personal computers (PCs) running the Linux operating system.
In 2009, a GPU-based (Graphics Processor Unit) computer system replaced the PC cluster, with the same computing power in only two nodes. Dedispersion is performed with a special filter in the complex Fourier domain. The timing resolution is a few 100 ns on the most stable millisecond pulsars (Cognard \& Theureau 2006; Cognard et al. 2009).

The NBPP was used to conduct a high-frequency, widebandwidth (1280-1430 MHz) pulsar survey of the Galactic plane from 1996 to 1998 (Foster et al. 1996). The search covered longitudes $-15^{\circ}<l<180^{\circ}$ for Galactic latitudes $|b|<3^{\circ}$, for a total sky coverage of 1170 square degrees. The survey was optimized to find distant pulsars with periods as short as the theoretical break-up speed of a neutron star. Over 40000 pointings were acquired, each with an integration time of $2 \mathrm{~min}$, for an expected $5 \sigma$ detection of a 0.5 mJy peak intensity profile after dedispersion. Observations were completed in late 1998. The final data volume is $\sim 5$ Tbytes.

A total of two pulsars were discovered in the survey, during the first 1998 data analysis based on a fast Fourier transform (FFT). Figures 1 and 2 show multiwavelength pulse profiles for PSRs J0248+6021 and J2240+5832, respectively. Table 1 lists various properties. PSR J0248+6021 has a spin period of $217 \mathrm{~ms}$, with a duty cycle of $12 \%$ at $1.4 \mathrm{GHz}$. A scattering tail is seen at low frequency, as expected from the large DM and confirmed by the observations made at higher frequencies. PSR J2240+5832 has a spin period of $140 \mathrm{~ms}$ and the radio peak is even narrower, $7 \%$ of a rotation at half-maximum. Relatively 


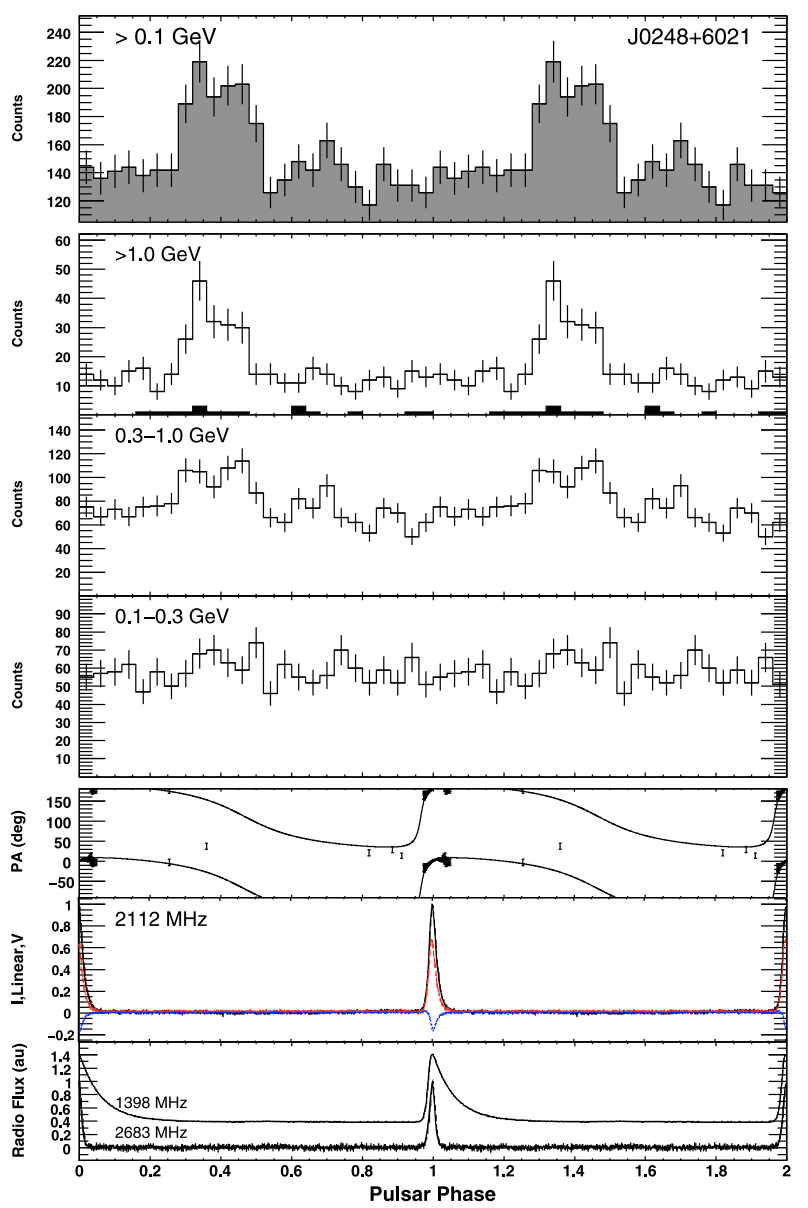

Fig. 1. Phase-aligned $\gamma$-ray and radio light curves for PSR J0248+6021 obtained with the Fermi Large Area Telescope and the Nançay Radio Telescope. The bottom panels show the radio profiles at three frequencies used to build the ephemeris. The second panel from the bottom shows the degree of linear (red dashed) and circular polarizations (blue dotted), as well as the linear polarization position angle and a Rotating Vector Model (RVM) fit. The other panels show the phase-folded $\gamma$-ray data in different energy bands. Two rotations are shown for clarity.

few pulsars are known in this direction $\left(l=106.6^{\circ}\right)$ and none with such a large $D M$. It may be one of the few pulsars known in the outer arm of the Galaxy and amongst the most distant known gamma-ray pulsars.

Subsequent FFT re-analysis using the PRESTO package developed by Ransom et al. (2003) identified no additional pulsars. We also used PRESTO to search for single pulses from the entire dataset: raw data were first dedispersed for 250 values ranging from 0 to $500 \mathrm{pc} \mathrm{cm}^{-3}$ keeping the original $60 \mu$ s resolution. Each time sequence was then searched for single pulses with a signal-to-noise ratio (hereafter $\mathrm{S} / \mathrm{N}$ ) greater than 5 . The search used matched filtering with boxcars up to 30 bins allowing good sensitivity up to $1.8 \mathrm{~ms}$ pulse width. We clearly detected series of very strong bursts from PSR J0248+6021 at the pulsar DM as shown in Fig. 4. No other single pulse signal was seen, including from PSR J2240+5832. PSR J0248+6021 is thus somewhat rare, in that it seems to burst in a way similar to PSR B0656+14 and RRATs (Rotating RAdio Transients, Weltevrede et al. 2006). The single pulse search is confirmed to be complementary to a classical FFT analysis, favoring pulsars with a low average signal strength but having strong bursts.

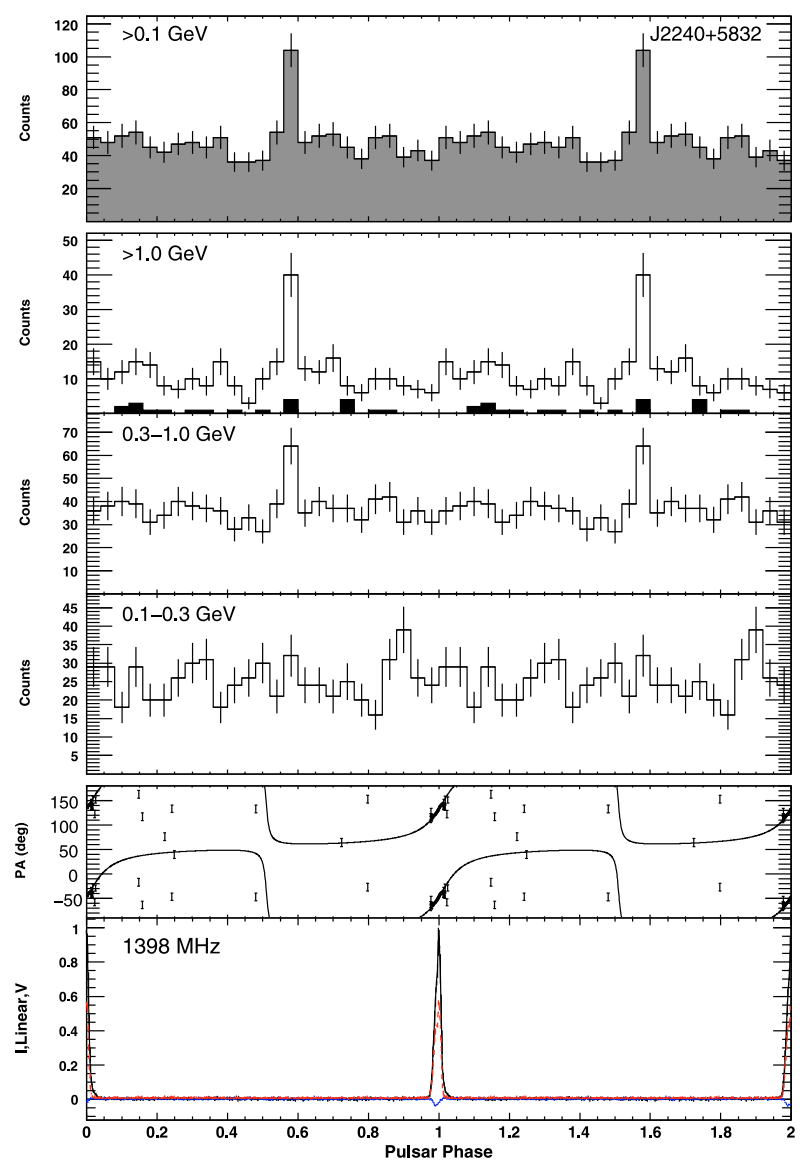

Fig. 2. Phase-aligned light curves for PSR J2240+5832. The bottom panel shows the $1.4 \mathrm{GHz}$ radio profile, along with the linear (red dashed) and circular polarizations (blue dotted). The second panel from the bottom shows the linear polarization position angle sweep with an RVM fit. The other panels show the phase-folded $\gamma$-ray data obtained with the Fermi Large Area Telescope in different energy bands. Two rotations are shown.

\section{Radio measurements}

\subsection{Flux density}

The radio flux for pulsar profiles obtained with the Nançay coherent dedispersion instrumentation can be accurately measured using a pulsed noise diode. The diode's counts-to-mJy scaling is obtained from fiducial galaxies. The diode is fired at $3.3 \mathrm{~Hz}$ for $15 \mathrm{~s}$ at the start of each observation, then phasefolded as for a pulsar. Several dozen observations at 1.4, 2.05, and $2.68 \mathrm{GHz}$ were selected for PSR J0248+6021. The $1.4 \mathrm{GHz}$ average value for the mean (i.e., phase-averaged) flux density of $13.7 \pm 2.7 \mathrm{mJy}$ is shown in Fig. 5, and a spectral index of $-1.19 \pm 0.07$ is obtained. These values are consistent with those obtained previously using the radiometer equation for both polarization channels, along with the known telescope characteristics $\left(T_{\text {sys }}=20.5 \mathrm{Jy}\right)$ and an assumed continuum flux of $6.5 \mathrm{Jy}$ at $1.4 \mathrm{GHz}$ in this sky direction (Reich 1982), to convert rms noise fluctuations in the off-pulse part of the profile to a Jansky scale.

Fourteen observations for PSR J2240+5832 yield a $1.4 \mathrm{GHz}$ average value for the mean flux density of $2.7 \pm 0.7 \mathrm{mJy}$, and a spectral index of $-1.83 \pm 0.25$, as also shown in Fig. 5. The sign convention for the radio energy spectral index is the opposite of that used for the gamma-ray photon index (i.e., $S(v) \propto v^{\alpha}$ as compared to $E^{-\Gamma}$ as in Eq. (2) in Sect. 5). 


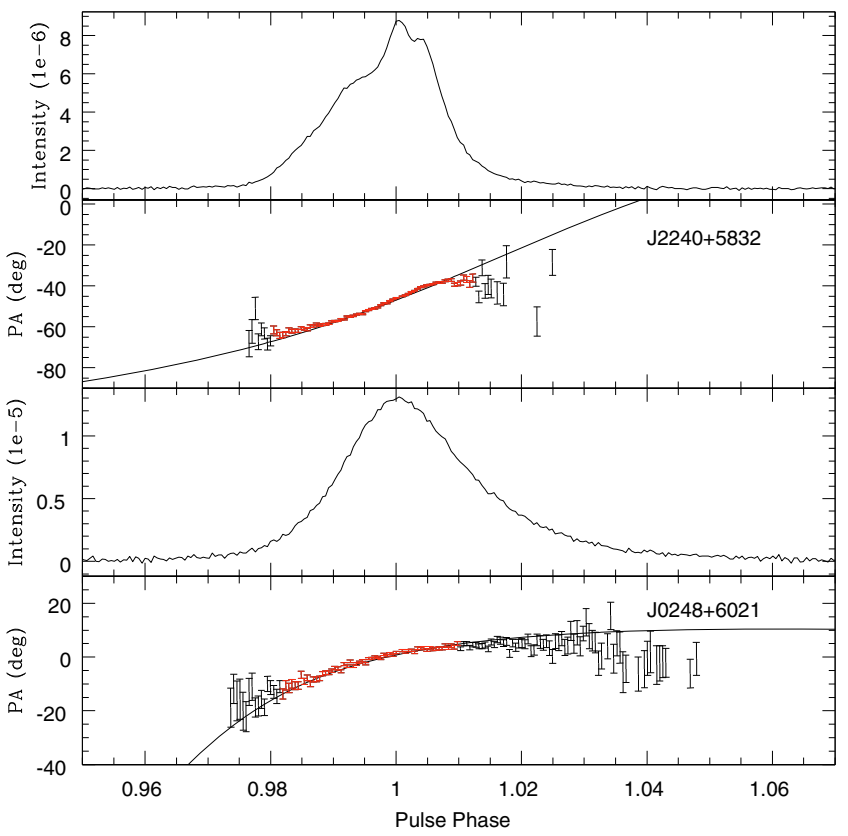

Fig. 3. Expanded view of the radio polarization position angle sweep near the peak in radio intensity. The red points show the data used in the RVM fit. The black points failed the selection cuts described in the text. Top two frames: for PSR J2240+5832, at $1.4 \mathrm{GHz}$. The RVM curve shown corresponds to inclination angles $\alpha=108^{\circ}$ and $\zeta=123^{\circ}$. Bottom two frames: for PSR J0248+6021, at $2.1 \mathrm{GHz}$. The RVM curve shown corresponds to inclination angles $\alpha=46^{\circ}$ and $\zeta=52^{\circ}$.
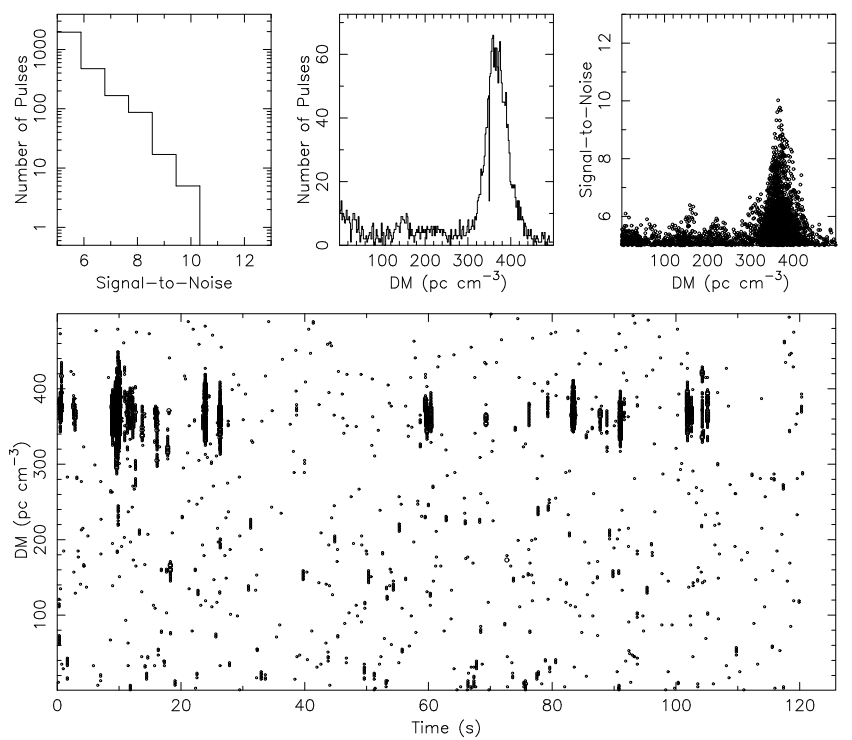

Fig. 4. A single pulse search of PSR J0248+6021. For each of $250 D M$ values, the 96 NBPP radio frequency samples are de-dispersed and summed. Bottom frame: summed intensities with signal-to-noise $5 \sigma$ above the average noise level are shown as points. Larger points indicate larger S/N. Top frames: projections showing a clear pulsar detection.

\subsection{Polarization}

Figure 3 zooms in on the sweep with phase of the linear polarization position angle (PA) for both pulsars. PA data is shown for all points with a minimum $\mathrm{S} / \mathrm{N}$ in Figs. 1 and 2 . We reduced the data using PSRCHIVE (Hotan et al. 2004). For PSR J0248+6021, we averaged $399 \mathrm{~min}$ of data from MJD 54851 to 55182 , and obtained a rotation measure of $R M=-158 \pm 6 \mathrm{rad} \mathrm{m}^{-2}$

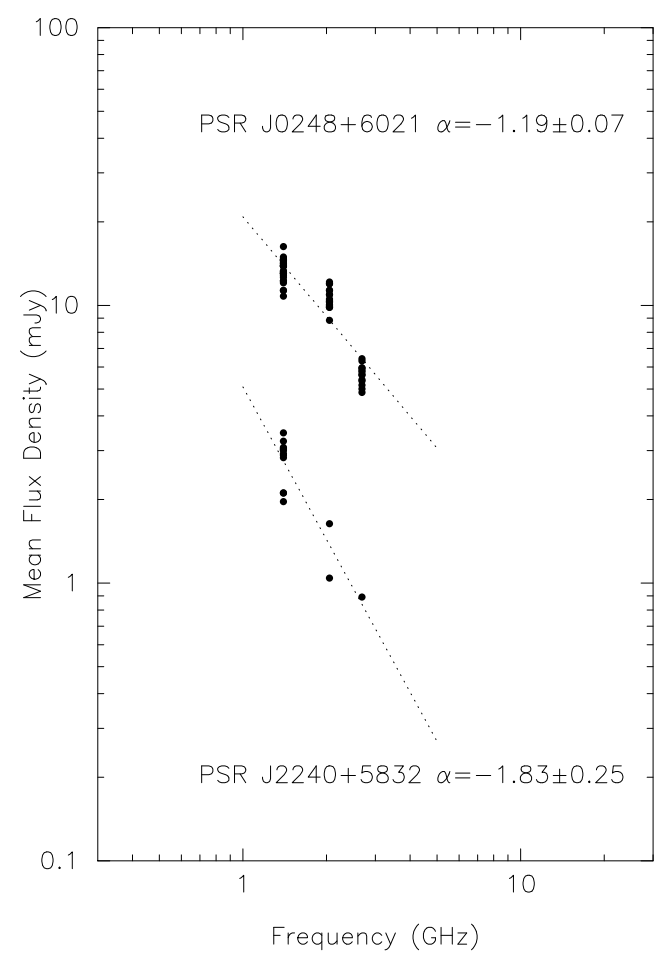

Fig. 5. Calibrated mean radio flux densities as a function of observation frequency. Note that the sign convention for the index is the opposite of that used for the gamma-ray spectra.

( $3 \sigma$ statistical uncertainties). At inflection, extrapolated to infinite frequency, we obtain $\mathrm{PA}_{0}=-67^{\circ} \pm 3^{\circ}$, shown in Fig. 6 together with the proper motion direction, discussed below. For PSR J2240+5832, we used 846 min of $1.4 \mathrm{GHz}$ data recorded from MJD 54866 to 55209 , yielding $R M=24 \pm 4 \mathrm{rad} \mathrm{m}^{-2}$ and $\mathrm{PA}_{0}=-39^{\circ} \pm 2^{\circ}$.

We fit the PA and phase data with the rotating vector model (RVM, Radhakrishnan \& Cooke 1969) to estimate the inclination $\zeta$ of the neutron-star rotation axis to the line-of-sight to Earth, and the angle $\alpha$ between the rotation axis and the magnetic axis. We use only data points with a minimum $\mathrm{S} / \mathrm{N}$ of $\sigma_{\mathrm{PA}}<2^{\circ}$. For PSR J0248+6021, the PA sweep inflection point seems to lead the radio peak due to bias induced by the broad scattering tail prominent at $1.4 \mathrm{GHz}$. We therefore use the $2.1 \mathrm{GHz}$ data for the RVM analysis. The fit results still vary with the choice of data in the tail but become stable when we favor the leading edge of the PA swing. We interpret this as a deformation due to residual scattering. We reject the remaining points with phase 0.01 beyond the peak. The results are robust once the choice to cut the tail is made. The points used for the RVM fit are shown in red in Fig. 3. As usual for RVM fits, the magnetic impact parameter $\beta=\zeta-\alpha$ is most strongly constrained, here to $\beta \approx+5^{\circ}$. A wide range of $\alpha$ from $40^{\circ}$ to $80^{\circ}$ provides comparably good fits, and acceptable fits extend from $\alpha \approx 25^{\circ}$ to $110^{\circ}$. The best fit has $\chi^{2}=86.6$ (reduced $\left.\chi^{2}=1.6\right)$.

The narrow radio pulse of PSR J2240+5832 is less affected by scattering: a fit to the $1.4 \mathrm{GHz}$ profile yields $\beta=16^{\circ}$ (for typical $\alpha$ ). Dispersion in the trailing data points causes a poor fit, with a minimum at $\chi^{2}=329$ (reduced $\chi^{2}=5.1$ ). Comparable fits persist from $\alpha \approx 75^{\circ}$ to $130^{\circ}$, and plausible solutions extend from $\alpha \approx 10^{\circ}$ to $150^{\circ}$.

The green contours in Figs. 7 and 8 summarize the RVM fit results, while the RVM curves in Fig. 3 show the fit for specific $(\alpha, \zeta)$ combinations within the favored region. These classic 

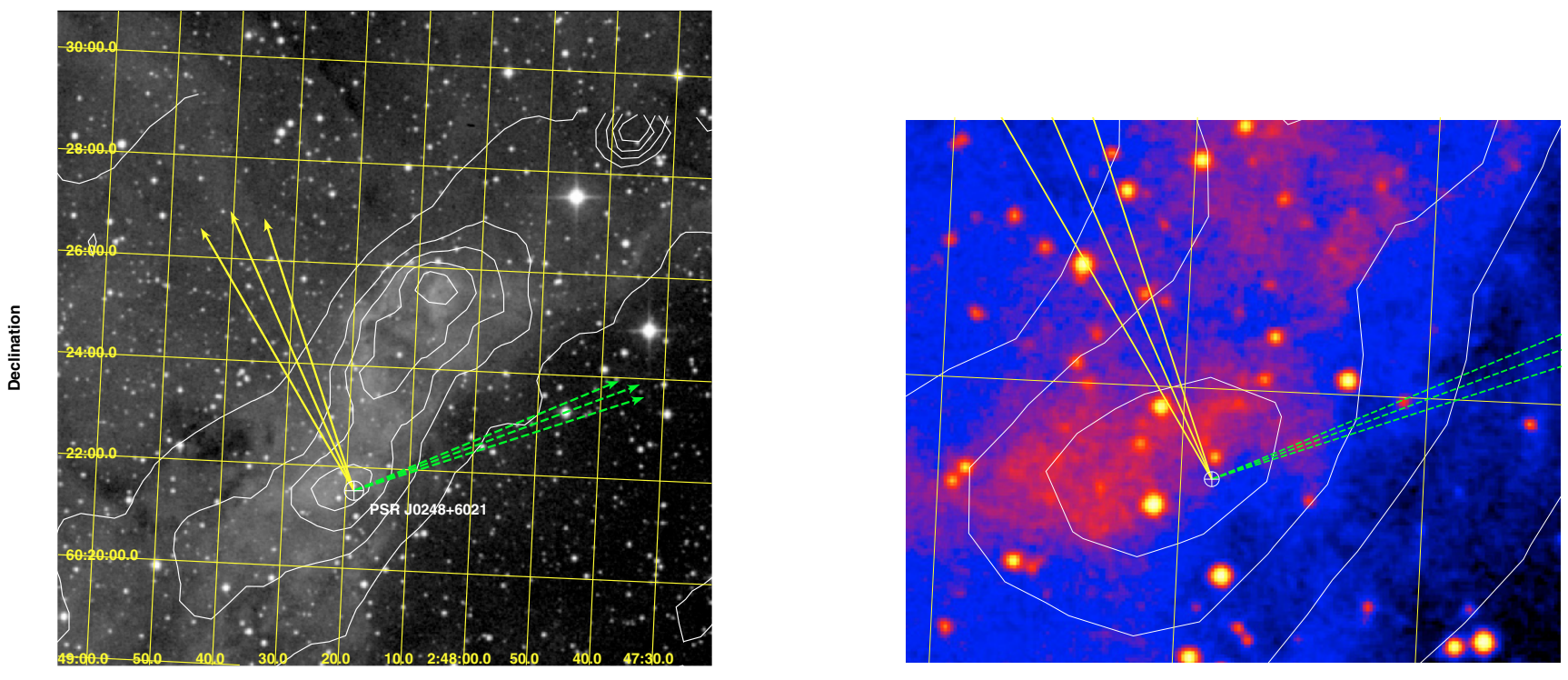

Fig. 6. Environment of PSR J0248+6021 from red Palomar Optical Sky Survey photographic plates (POSS-II, background) and NRAO VLA Sky Survey (NVSS) $1.4 \mathrm{GHz}$ charts (contours). The solid arrows show the proper-motion position angle and uncertainty. The dashed arrows show the polarization position angle at the inflection point and uncertainty, extrapolated to infinite frequency. The arrow lengths have no meaning. The zoomed image at right shows the star near the pulsar excluded as an optical counterpart, as well as the optical arc emanating from the star discussed in the text.
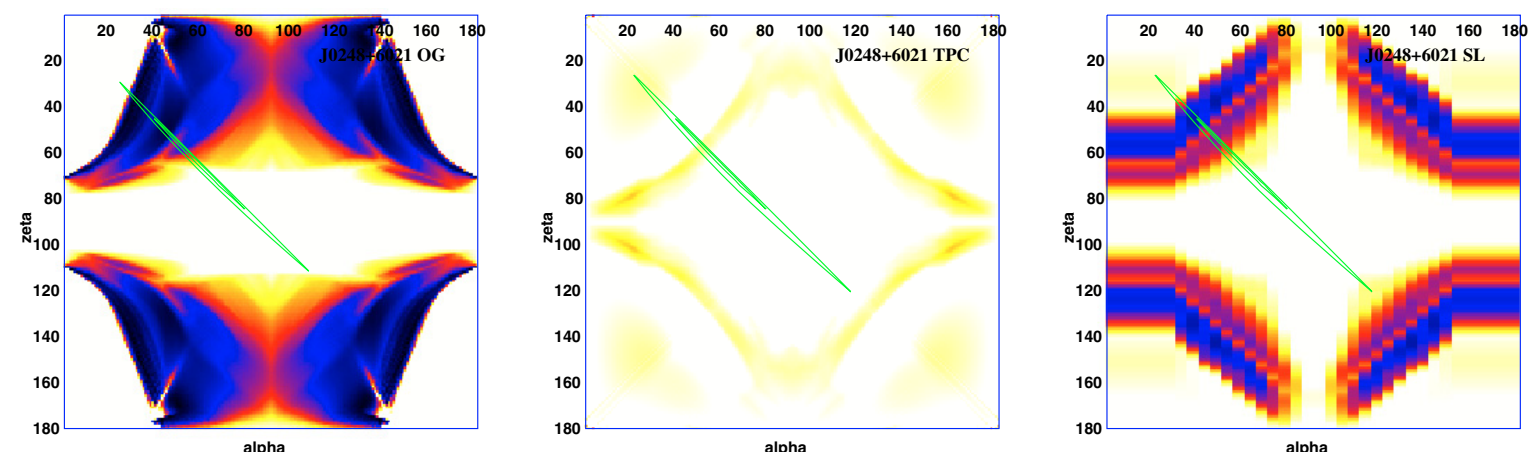

alpha

Fig. 7. Pulsar geometry and emission modeling for PSR J0248+6021. Green contours show the rotating vector model fit to the radio polarization data (contours at $\delta\left(\chi^{2} /\right.$ d.o.f. $)=+0.25,+0.5$ above the minimum $\chi^{2} /$ d.o.f. of 1.6$)$. The color backgrounds are $\chi_{3}$ maps of the fit to the observed $>100 \mathrm{MeV}$ pulse profile to the outer gap model (left), the two-pole caustic model (middle), and the separatrix layer model (right), for different values of the magnetic inclination, $\alpha$, and the minimum angle to the line-of-sight, $\zeta$ (Romani $\&$ Watters 2010). Each panel has the same color scale, where dark colors represent better fits. The preferred models lie along the green RVM-selected band.

RVM fits assume radio emission at low altitudes. It is becoming increasingly clear (Karastergiou \& Johnston 2007; Ravi et al. 2010) that for many young pulsars, especially the gamma-ray detected pulsars, the radio emission arises at a substantial fraction of the light cylinder radius, $R_{\mathrm{LC}}$. This in turn means that the polarization PA sweeps are offset significantly from the radio pulse (Blaskiewicz et al. 1991), and that more subtle distortions in the PA sweep shape may occur. Self-consistent fitting using large radio altitudes should therefore lead to a shift in the inferred $\alpha$ and $\zeta$ values.

\subsection{Radio timing and proper motions}

Both pulsars were observed regularly for twelve years with increasingly advanced instrumentation, except for 18 months in 2003-2004 when only the BON prototype with a $16 \mathrm{MHz}$ bandwidth was available. Figure 9 shows the evolution of the timing precision over the years, and also indicates five principal glitch epochs for PSR J0248+2240. The largest was $\delta v / v=$ $7.5 \times 10^{-7}$ in March 2009 (MJD 54 897.41), where $v$ is the rotation frequency and $\delta v$ is the permanent pulse frequency increment. The epochs and $\delta v / v$ values for the other four glitches were $\left(52240.0,1.6 \times 10^{-10}\right),\left(52850.0,8.2 \times 10^{-9}\right),(53700.0$, $\left.6.9 \times 10^{-11}\right)$ and $\left(53900.0,-2.6 \times 10^{-11}\right)$. Figure 10 shows the BON data, including the data used to phase-fold the gamma-ray photons, in greater detail. The timing model includes a first time derivative of the $D M$ ("DM1" variable in TEMPO2). Higherorder $D M$ variations as well as achromatic timing noise can be seen. For PSR J2240+5832, no large glitches were seen, but even after whitening some residual structure due to timing noise remains.

Timing noise biases proper-motion measurement unless care is taken. Different approaches were compared, summarized in 

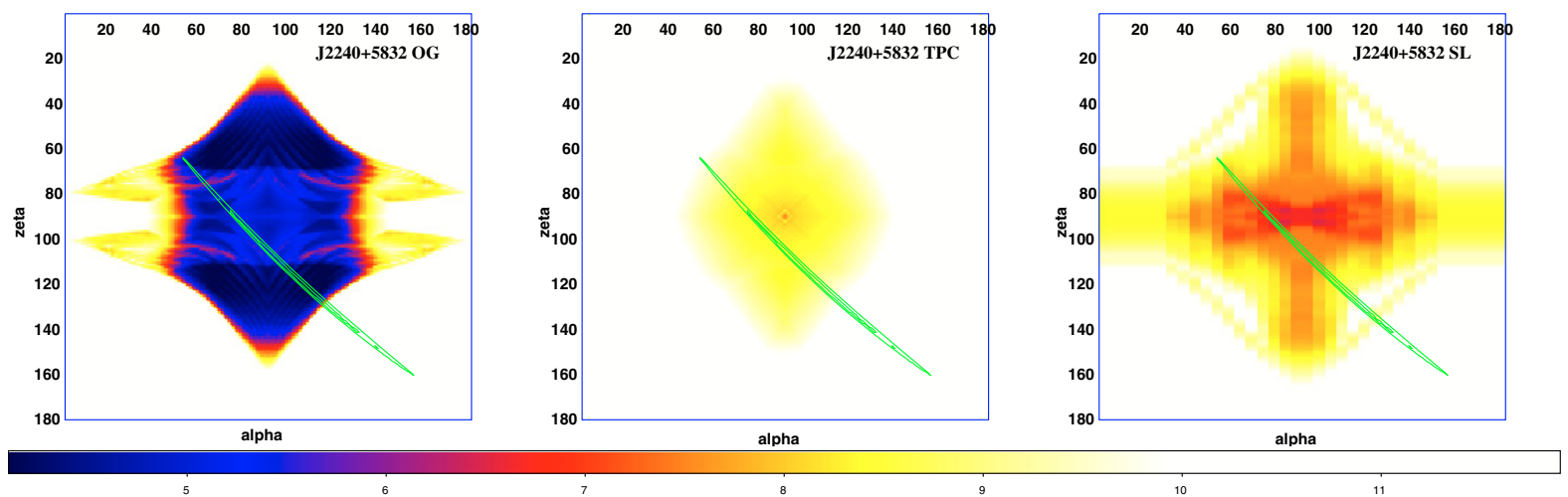

Fig. 8. Pulsar geometry and emission modeling for PSR J2240 +5832 , as in Fig. 7. The green contours of the RVM fits lie $\delta\left(\chi^{2} /\right.$ d.o.f. $)=+0.4,+0.8$ above the minimum of $\chi^{2} /$ d.o.f. $=5.1$.

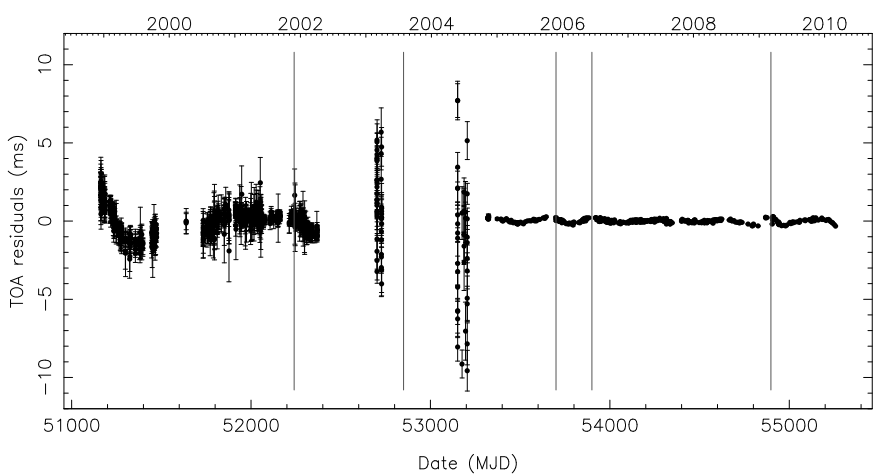

Fig. 9. Time-of-arrival post-fit residuals for PSR J0248+6021 when glitch parameters are fit. Vertical lines indicate glitch epochs. The timing resolution of the datasets using the NBPP (left), BON prototype (middle), and $\mathrm{BON}$ (right) backends is apparent.

Fig. 11, where the labels for the methods are described below. The first two methods compare the position at different epochs with separate data spans, fitting for rotational parameters $(v, \dot{v}, \ddot{v})$ and pulsar position. The old NBPP timing data yield a position at MJD 51250 , with another position at MJD 53900 from the BON data, for a proper motion of $\mu_{\alpha}=13.4 \pm 8{\text { mas } \mathrm{yr}^{-1} \text { and }}^{-1}$

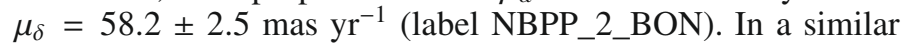
way, the BON data were divided into two equal parts. Two positions obtained at MJDs 53700 and 54500 indicate a proper

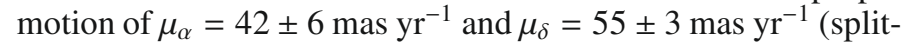
ted_BON). The two last methods use the harmonic whitening procedure proposed by Hobbs et al. (2004) in their Appendix A. An iterative process using a fit of harmonically related sinusoids ("FITWAVE" in TEMPO2, Edwards et al. 2006) is applied following a strict sequence of different parameter adjustments. This procedure was applied to both the old NBPP and the new BON timing data. The NBPP data give $\mu_{\alpha}=24 \pm 30$ mas $\mathrm{yr}^{-1}$

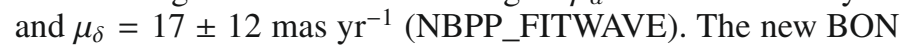
timing data imply a proper motion of $\mu_{\alpha}=62 \pm 3 \mathrm{mas} \mathrm{yr}^{-1}$ and $\mu_{\delta}=35.6 \pm 1.8 \mathrm{mas} \mathrm{yr}^{-1}$ (BON_FITWAVE).

The error bars shown in Fig. 11 for the successive measurements sometimes do not overlap because systematic biases due to e.g., timing noise or covariances underestimated by the fitting routines, are larger than the statistical uncertainties. We computed a weighted mean proper motion for PSR J0248+6021 based on all the determinations except the NBPP_FITWAVE, which has very large uncertainty and hence constrains little. The

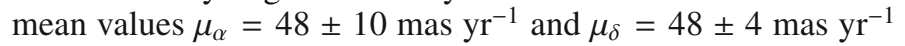

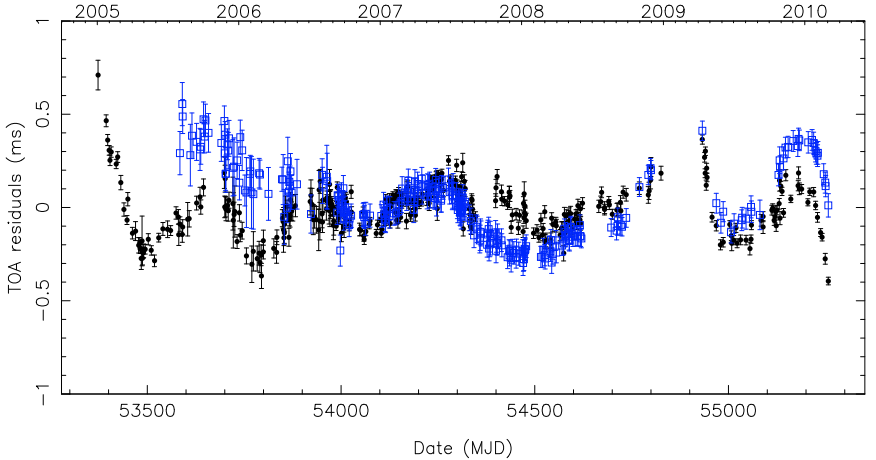

Fig. 10. Time-of-arrival post-fit residuals for PSR J0248+6021, allowing for linear variations of dispersion measure over time, as in Fig. 9, but showing only data acquired with the BON backend. The $2.0 \mathrm{GHz}$ data (blue squares) are clearly above the $1.4 \mathrm{GHz}$ data (black dots) for the year 2006 and for 2009-2010, but below these data in 2008. We interpret the increasing, then decreasing $D M$ value as evidence that the neutron star is moving through a dense environment.

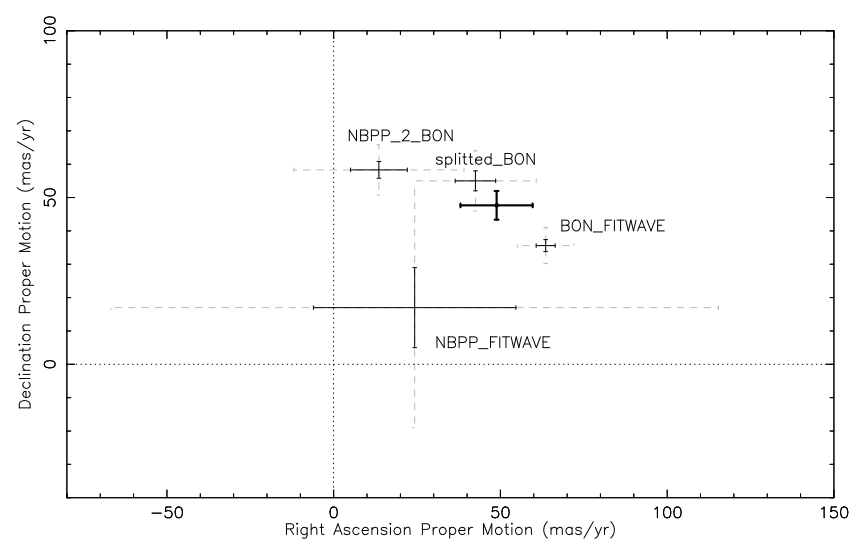

Fig. 11. The PSR J0248+6021 proper motion measured by different methods (Sect. 3.3). The weighted average of $\mu=\sqrt{\mu_{\alpha}^{2} \cos ^{2}(\delta)+\mu_{\delta}^{2}}=$

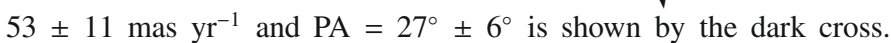
The three different determinations are labeled NBPP_2_BON, splitted_BON, and BON_FITWAVE and are described in the text. Solid error bars are $\pm 1 \sigma$ and dashed error bars are $\pm 3 \sigma$.

were obtained by weighting by the inverse of the uncertainties. The total is $\mu=\sqrt{\mu_{\alpha}^{2} \cos ^{2}(\delta)+\mu_{\delta}^{2}}=53 \pm 11 \mathrm{mas} \mathrm{yr}^{-1}$ and the celestial position angle is $\mathrm{PA}=\tan ^{-1}\left(\mu_{\alpha} \cos (\delta) / \mu_{\delta}\right)=27^{\circ} \pm 6^{\circ}$. 
PSR J2240+5832's proper motion is roughly half that of the other pulsar. The measured value became stable only after multiple iterations of the method cited above (Hobbs et al. 2004), apparently due to one or two marginally detectable glitches that

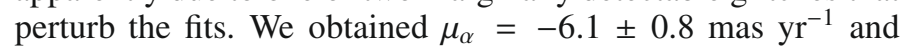
$\mu_{\delta}=-21.0 \pm 0.4 \operatorname{mas}_{\mathrm{yr}^{-1}}\left(\mu=\sqrt{\mu_{\alpha}^{2} \cos ^{2}(\delta)+\mu_{\delta}^{2}}=21.3 \pm\right.$

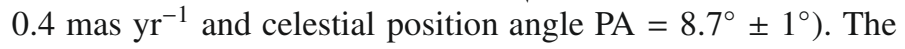
uncertainties are statistical. To be conservative, we assume systematic uncertainties of the same magnitude as those observed for PSR J0248+6021, and thus obtain $\mu=21 \pm 4 \mathrm{mas} \mathrm{yr}^{-1}$ (same fractional error) and celestial position angle $\mathrm{PA}=9^{\circ} \pm 6^{\circ}$ (same absolute value).

Figure 6 shows both the proper motion and polarization position angles for PSR J0248+6021. They are roughly perpendicular (difference of $94^{\circ} \pm 7^{\circ}$ ). Johnston et al. (2007) recall that neutron star velocity vectors and spin-axis may naturally become aligned at birth, if the supernova "kick" is preferentially along the spin-axis, and the radio emission is orthogonal to the magnetic field. That paper adds a new sample of measurements for which this scenario appears to hold about half the time, weakening the observational evidence supporting this picture. For PSR J2240+5832, the difference between the velocity and polarization PA's is $48^{\circ} \pm 6^{\circ}$ : hence, in this new sample of two pulsars, again only half seem to comply with the "rule".

\section{Distances and surroundings}

One goal of this work is to compare model predictions of the pulsars' gamma-ray luminosity $L_{\gamma}$ with observations. Following the "Atlas" of Watters et al. (2009) and its update (Romani \& Watters 2010), we define

$L_{\gamma}=4 \pi f_{\Omega} G_{100} d^{2}$

for $G_{100}$, the integral gamma-ray energy flux above $100 \mathrm{MeV}$. The beam correction factor $f_{\Omega}$ is the ratio of the gamma-ray flux radiated into all space to that along the line-of-sight, both averaged over a neutron star rotation. Most models depend on the inclination and viewing angles $\alpha, \zeta$ as discussed below. The distance $d$ is clearly important.

\subsection{Dispersion measure, kick velocities, and rotation measures}

\subsubsection{Distance to PSR J0248+6021}

As stated earlier, the DM for PSR J0248+6021 is so large for this line-of-sight $\left(l=136.9^{\circ}, b=0.698^{\circ}\right)$ that the NE2001 model places it outside the Galaxy. However, Fig. 6 shows extensive clouds that add to the electron column density and bias the NE2001 distance.

The pulsar lies $0.3^{\circ}$ west of the center of the open cluster IC 1848, which itself lies within the giant HII region W5, the "Soul" nebula in the Galaxy's Perseus arm. The cluster diameter is $0.34^{\circ}$ (Kharchenko et al. 2005). The pulsar was born $\mu \tau_{\mathrm{c}} \lesssim 1^{\circ}$ southwest of its current position. The heliocentric distance to IC 1848 estimated from both main sequence fitting of the star cluster and a Galactic kinematic model converges towards $2 \mathrm{kpc}$ (Kharchenko et al. 2005). That reference gives no distance uncertainty ${ }^{1}$. Distance uncertainties for individual stars from other studies, e.g. using photometric measurements

\footnotetext{
${ }^{1}$ See also WEBDA database, http: //www. univie.ac . at/webda/
}

(Morgan et al. 2009) do not exceed $\pm 0.4 \mathrm{kpc}$, and we assume $\pm 0.2 \mathrm{kpc}$. We now argue that the pulsar is at the distance of the open cluster, i.e., on the near side of W5.

The parallel component of the magnetic field along the lineof-sight to PSR J0248+6021 is $B_{\|}=1.23 R M / D M=-0.5 \mu \mathrm{G}$. This matches nicely the expectation from Fig. 6 of Mitra et al. (2003) and therefore the pulsar is most likely within the Perseus arm. From Reid et al. (2009), the far edge of that arm is $<3.6$ kpc distant.

The proper motion of PSR J0248+6021 is, with its uncertainty, $>42$ mas $\mathrm{yr}^{-1}$. The largest measured neutron-star velocities due to supernova "kicks" at birth are of the order of $1000 \mathrm{~km} \mathrm{~s}^{-1}$ (Hobbs et al. 2005). Thus, if PSR J0248+6021 were beyond $4.8 \mathrm{kpc}$ it would have an unusually large kick velocity. Conversely, a $2 \mathrm{kpc}$ distance implies a very typical transverse velocity of $v_{\mathrm{T}} \sim 500 \mathrm{~km} \mathrm{~s}^{-1}$. Furthermore, the $D M$ of PSR J0248+6021 increased steadily by $0.3 \mathrm{~cm}^{-3}$ pc per year from 2006 to 2008, and decreased at the same rate in 2009 and 2010. Figure 10 shows that the detailed behavior is complex. The simplest interpretation of the $D M$ evolution is that the pulsar is moving rapidly in a dense environment, that is, that the pulsar is in a cloud (conceivably a nearer, invisible cloud).

For the cloud coincident with the pulsar (Fig. 6), the largest velocity (largest Doppler shift) of reliable HI features towards PSR J0248+6021 is $-50 \mathrm{~km} \mathrm{~s}^{-1}$. In light of the known velocity reversal in this part of the Perseus arm (Tian et al. 2010) the cloud is thus between 2 and $3 \mathrm{kpc}$, like the rest of W5. High resolution $\mathrm{H} \alpha$ imaging could confirm this preliminary indication.

Towards PSR J0248+6021, the NE2001 model places the edge of the stellar Galactic disk $9 \mathrm{kpc}$ from the Sun, with a $D M$ of $210 \mathrm{~cm}^{-3} \mathrm{pc}$. A dense local environment, as within W5, may explain the large observed electron column density $D M=$ $370 \mathrm{~cm}^{-3}$ pc. For $2 \mathrm{kpc}$, NE2001 predicts $D M \simeq 61 \mathrm{~cm}^{-3} \mathrm{pc}$, implying an excess of $\sim 315 \mathrm{~cm}^{-3} \mathrm{pc}$. The main part of the HII region neighboring the pulsar is a shell of diameter $0.5^{\circ}$ on the sky, equivalent to a true size of $17.5 \mathrm{pc}$ at $2 \mathrm{kpc}$. This converts into an electron density in the cloud of $\left\langle n_{\mathrm{e}}\right\rangle \simeq 18 \mathrm{~cm}^{-3}$. Though large, this is typical of other HII regions, where values from 0.1 to $100 \mathrm{~cm}^{-3}$ are seen (see e.g. Mitra \& Ramachandran 2001, for their study of the scatter broadening in the direction of the Gum Nebula). The WISE collaboration (Wright 2010) released a detailed image of the Heart and Soul nebulae seen between 3.4 and 22 microns $^{2}$. PSR J0248+6021 lies in one of the "thickest" areas, where the shell is seen edge-on and the electron density is surely greater than the average value, indicating again that the large $D M$ is compatible with a small pulsar distance.

The large scattering tail excludes the notion that PSR J0248+6021 may be within the Orion spur, thus only $\sim 1 \mathrm{kpc}$ away, because of nearby but invisible electron clouds. To conclude, PSR J0248+6021 is almost certainly in W5, at the distance of IC 1848, and we use $d=2.0 \pm 0.2 \mathrm{kpc}$.

\subsubsection{Distance to PSR J2240+5832}

PSR J2240+5832 has $D M=263.5 \mathrm{~cm}^{-3} \mathrm{pc}$ and along this lineof-sight $\left(l=106.57^{\circ}, b=-0.111^{\circ}\right)$ the NE2001 distance is $10.3_{-3.3}^{+\infty} \mathrm{kpc}$. Archival optical and radio images show no bright structures near the pulsar position that would indicate significant deviations from the NE2001 maps. However, the pitch angle of the outer arm has been shown to be smaller than known when the NE2001 model was created (Reid et al. 2009), and the far edge of the outer arm is $<8.3 \mathrm{kpc}$ in this direction.

2 http://antwrp.gsfc.nasa.gov/apod/ap100601.html 
Again assuming a transverse velocity $v_{\mathrm{T}}<1000 \mathrm{~km} \mathrm{~s}^{-1}$,

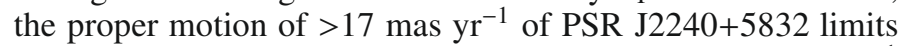
its distance to $<12 \mathrm{kpc}$. Conversely, a value of $v_{\mathrm{T}}=400 \mathrm{~km} \mathrm{~s}^{-1}$ would place the pulsar in the Perseus arm, which extends from 3 to $4.6 \mathrm{kpc}$ in this direction. PSR J2240+5832 is therefore among the few pulsars in this direction for which rotation measures are available (Han et al. 2006). The positive value of $R M=$ $24 \mathrm{rad} \mathrm{m}^{-2}$, albeit small, is anomalous: other low-latitude $R M$ 's for $90^{\circ}<l<120^{\circ}$ are negative. On the other hand, in this direction no other pulsar between 6 and $8 \mathrm{kpc}$ has an $R M$ measurement and the positive value may be due to the magnetic field inversion beyond the Perseus arm suggested by Han et al. (2006). The magnetic field $B_{\|}=1.23 R M / D M=0.1 \mu \mathrm{G}$ is within the range of observed values in Mitra et al. (2003).

Neutron stars are most likely born within arms and, since $v_{\mathrm{T}} \tau_{\mathrm{c}}<0.1 \mathrm{kpc}$, the pulsar should not have gone far from its birthplace. We conclude by presenting two possible values for the distance to PSR J2240+5832: one in the outer $\operatorname{arm}(d=7.7 \pm$ $0.7 \mathrm{kpc})$ and another in the Perseus arm $(d=3.8 \pm 0.8 \mathrm{kpc})$. If in the outer arm, $v_{\mathrm{T}}$ is large but not exceptional and the pulsar is farther than most known gamma-ray pulsars. If it is in the Perseus arm, the $D M$ excess and positive $R M$ remain unexplained but the speed and distance are more typical. We see below that the two values of $L_{\gamma}$ obtained using the two distances are both plausible. VLBI astrometric parallax measurements would be of great interest for these and all gamma-ray pulsars.

\subsection{Evidence of a pulsar wind nebula?}

PSR J0248+6021 coincides with the heart of an elongated nodule 15' long (see Fig. 6), seen at both $21 \mathrm{~cm}$ (NVSS, Condon et al. 1998) and in the optical. The pulsar proper motion is $\sim 45^{\circ}$ from the nodule long axis. The POSS-II (Palomar Optical Sky Survey) image shows an arc $20^{\prime \prime}$ long about $5^{\prime \prime}$ from the pulsar position. The simple formulae for the size and offsets for pulsar wind nebula (PWN) termination shocks given by Kargaltsev \& Pavlov (2008) are roughly consistent with what we observe. Might we be seeing shocks in the interstellar medium caused by the pulsar's high transverse velocity?

To explore the idea of a possible PWN, we obtained Swift (Gehrels et al. 2004) observations of PSR J0248+6021 with data taken over two days, 2009 Dec. 10-11. The target was not detected in the X-ray Telescope (XRT; Burrows et al. 2005) combined $7.2 \mathrm{ks}$ exposure and we derive a $3 \sigma$ limit on the source count rate $(0.5-8 \mathrm{keV})$ of $0.0024 \mathrm{cts} / \mathrm{s}$ after dead time, vignetting, and PSF corrections. Using PIMMS, this corresponds to an observed flux limit of $1.4 \times 10^{-13} \mathrm{erg} \mathrm{cm}^{-2} \mathrm{~s}^{-1}$, or $2.0 \times$ $10^{-13} \mathrm{erg} \mathrm{cm}^{-2} \mathrm{~s}^{-1}$ unabsorbed with Galactic $N_{\mathrm{H}}=8.3 \times$ $10^{21} \mathrm{~cm}^{-2}$ (Kalberla et al. 2005), assuming a power-law photon flux with $\Gamma=1.5$ as is typical among observed X-ray PWN (same sign convention for $\Gamma$ as in Eq. (2), Sect. 5). For $d=2 \mathrm{kpc}$, we obtain (absorbed) $L_{\mathrm{X}}<6 \times 10^{31} \mathrm{erg} / \mathrm{s}$ for the PWN X-ray luminosity. Only eight of the 40 X-ray PWN's in Kargaltsev \& Pavlov (2008) are dimmer: if there is an X-ray PWN, it is faint, possible for a PWN of the age of these pulsars.

An upper limit on GeV PWN emission is given in Sect. 5.1. The position of PSR J0248+6021 is at the corner of both the MAGIC (Albert et al. 2006) and the VERITAS (Acciari et al. 2008) $\mathrm{TeV}$ significance maps for $\mathrm{LS} \mathrm{I}+61^{\circ} 303$. No evidence of $\mathrm{TeV}$ emission from a putative PWN is apparent. Reanalysis of the complete $\mathrm{TeV}$ datasets optimized for a source at this location with PWN-like properties (i.e. possibly offset, and/or spatially extended) is encouraged.

\subsection{Upper limit for detection of an optical pulsar}

Digital Sky Survey images show a faint source seemingly coincident with the pulsar position. Analyzing the Ultra-Violet/Optical Telescope (UVOT; Roming et al. 2005) $U$-filter image (exposure: 3575 s) obtained with Swift on 2009 December 11th, the faint source is detected at $6.0 \sigma$. We measure its center (J2000) at RA $=02^{\mathrm{h}} 48^{\prime} 18.711^{\prime \prime}$, dec $=60^{\mathrm{d}} 21^{\prime} 38.98^{\prime \prime}$ with a statistical uncertainty of $0.46^{\prime \prime}$ (90\% confidence). Its observed flux density is $1.07 \pm 0.18 \times 10^{-2} \mathrm{mJy}$ at $8.56 \times 10^{14} \mathrm{~Hz}$ corresponding to magnitude $20.33 \pm 0.18$. The uncertainties are statistical. This source is not detected in the UVOT W1-filter image (3537 s) obtained on 2009 December 10th and we derive a $3 \sigma$ upper limit of $<2.80 \times 10^{-3} \mathrm{mJy}$ at $1.14 \times 10^{15} \mathrm{~Hz}$. Pulsar timing is incompatible with the position of the optical object and there is therefore no evidence of an optical pulsar.

\section{Gamma-ray observations}

The LAT is an electron-positron pair conversion telescope that was placed in orbit with the Fermi satellite on 2008 June 11 (Atwood et al. 2009; Abdo et al. 2009e). The LAT covers the $20 \mathrm{MeV}$ to $>300 \mathrm{GeV}$ energy range with higher sensitivity and more accurate localisation than previous instruments (an on-axis effective area $\sim 8000 \mathrm{~cm}^{2}$ above $1 \mathrm{GeV}$ and angular resolution $\theta_{68} \sim 0.8^{\circ}$ at $\left.1 \mathrm{GeV}\right)$. LAT measurements yielded a catalog of 1451 gamma-ray "1FGL" sources during its first year (Abdo et al. 2010a).

We used the standard Science Tools software package for Fermi LAT data analysis ${ }^{3}$. For both pulsars, we selected data collected between 2008 August 4 (MJD 54 682) when Fermi began scanning-mode operations, and the end of the ephemeris validity range (over 15 months in both cases). We kept "diffuse" class events (highest probability of being $\gamma$-ray photons) within a $15^{\circ}$ "region-of-interest" (ROI) around the pulsar. We excluded events with zenith angles $>105^{\circ}$ to reject the $\gamma$-ray albedo from the Earth's limb. Photon phases were calculated using the TEMPO2 pulsar timing software (Edwards et al. 2006).

At low photon energies, multiple scattering dominates the LAT's angular resolution. For the gamma-ray pulse profiles (phase histograms), we applied an energy-dependent angular radius cut centered on the pulsar that approximates the instrument point spread function (PSF), given by $\left\langle\theta_{68}(E)\right\rangle=\sqrt{\left(5.12^{\circ}\right)^{2}(100 \mathrm{MeV} / E)^{1.6}+\left(0.07^{\circ}\right)^{2}}$, which corresponds to a $68 \%$ containment angle (P6_V3 Instrument Response Function, “IRF”). A maximum radius of $\theta_{68}^{\max }=0.8^{\circ}$ and $0.7^{\circ}$ for PSRs $\mathrm{J} 0248+6021$ and $\mathrm{J} 2240+5832$, respectively, reduces the background at low energies. We also remove events from nearby 1 FGL sources within $<3^{\circ}$ using the same bounded, energy-dependent radius as applied to the pulsar.

The on-pulse spectra were obtained with a maximum likelihood analysis (Mattox et al. 1996) of the LAT data within $15^{\circ}$ of the pulsar, using the Fermi science tool "gtlike". The likelihood method weights events from the target and background sources according to the energy-dependent PSF, which explains the need for such a large ROI. We excluded time intervals when the ROI intersected the Earth's limb. In this paper, we modeled the pulsars using the functional form

$$
\frac{\mathrm{d} N}{\mathrm{~d} E}=N_{0} E^{-\Gamma} \exp \left[-\left(\frac{E}{E_{\mathrm{c}}}\right)^{\beta}\right] \mathrm{cm}^{-2} \mathrm{~s}^{-1} \mathrm{MeV}^{-1},
$$

${ }^{3}$ Gamma-ray data, analysis software, rotation ephemerides, and the diffuse background models are publically available at the Fermi Science Support Center, FSSC, http://fermi.gsfc.nasa.gov/ssc/ 
where $N_{0}$ is the differential flux normalization (ph $\mathrm{cm}^{-2} \mathrm{~s}^{-1} \mathrm{MeV}^{-1}$ ), $\Gamma$ the photon index, and $E_{\mathrm{c}}$ the cutoff energy. A pure power law $(\beta=0)$ describes many gamma-ray sources, such as active galactic nuclei (Abdo et al. 2010a). The 46 gamma-ray pulsars discussed in Abdo et al. (2010b) are generally well-described by a simple exponential cutoff, $\beta=1$, a shape predicted by outer magnetosphere emission models (see the Discussion, below). Models where gamma-ray emission occurs closer to the neutron star can have sharper "super-exponential" cutoffs, e.g. $\beta=2$.

The Galactic diffuse emission was modeled using the gll_iem_v02 map cube based on six Galactocentric "ring" maps of $N\left(\mathrm{H}_{I}\right)$ and $W(\mathrm{CO})$ and on the spatial distribution of the inverse Compton intensity modeled by GALPROP (Strong et al. 2004a,b). Diffuse extragalactic gamma-ray emission and residual instrument backgrounds were modeled jointly by the isotropic component isotropic_iem_v02. Both models are available from the FSSC. All 1FGL sources within $20^{\circ}$ were modeled with a power-law. Sources farther than $5^{\circ}$ from the target pulsar were assigned fixed power law spectra, with parameters taken from the 1FGL source catalog. Spectral parameters for the pulsars and sources within $5^{\circ}$ of the pulsar were allowed to vary. Systematic uncertainties were estimated by reapplying the fitting procedures using bracketing IRFs where the effective area was shifted (linear extrapolations in log space) by $\pm 10 \%$ at $0.1 \mathrm{GeV}$, $\pm 5 \%$ near $0.5 \mathrm{GeV}$, and $\pm 20 \%$ at $10 \mathrm{GeV}$.

\subsection{PSR J0248+6021}

PSR J0248+6021 is called 1FGL J0248.3+6021 in the LAT catalog (Abdo et al. 2010a). It is in the Galactic plane, $1.3^{\circ}$ away from the bright gamma-ray source 1FGL J0240.5+6113, identified as the Be star binary LSI $+61^{\circ} 303$ (Abdo et al. 2009 d). No other 1 FGL sources are within $3^{\circ}$. The high $\dot{E}$ pulsar PSR J0205+6449 in 3C 58 is about $6^{\circ}$ away (Abdo et al. 2009b). PSR J0248+6021 was discovered towards the end of EGRET's lifetime, and ephemerides contemporaneous with only a fraction of EGRET data exist. Before the Fermi mission, we searched for gamma-ray pulsations in all EGRET data for a range of $(v, \dot{v})$ values extrapolated from our radio ephemeris. However, no pulsed signal was detected presumably because of the poor $\mathrm{S} / \mathrm{N}$ of the EGRET data.

Fermi LAT data for PSR J0248+6021 do however exhibit clear pulsations for the 3810 photons with energies $>0.1 \mathrm{GeV}$ remaining after cuts. Figure 1 shows the folded light curve of these events for different energy bands, along with the radio profiles used to derive the timing parameters. The light curve in each energy interval consists of one broad peak between 0.20 and 0.55 in phase, which dominates the highest energy band $(>1 \mathrm{GeV})$ but is progressively less pronounced with decreasing energy. The pulsation significances based on the binindependent $H$-test (de Jager \& Büsching 2010) are $\sim 1 \sigma, 6.5 \sigma$, $8.2 \sigma$, respectively, for the three energy bands $0.1-0.3,0.3-1.0$, and $>1 \mathrm{GeV}$. Between 0.3 and $1 \mathrm{GeV}$, a structure with statistical significance of $\sim 3 \sigma$ (signal/ $\sqrt{\text { background }}$ ) appears between phases 0.6 and 0.7 . However, it is not observed in the other energy bands, and a full likelihood analysis in that phase interval yields an excess with a significance of only $2 \sigma$ between 0.1 and $100 \mathrm{GeV}$. We fit the main $\gamma$-ray peak $(0.2<\phi<0.55)$ above $0.1 \mathrm{GeV}$ with two half-Lorentzian functions. This shape provides a higher value of $\chi^{2}$ than others and accommodates differences between the leading and trailing edges. The fit peak is
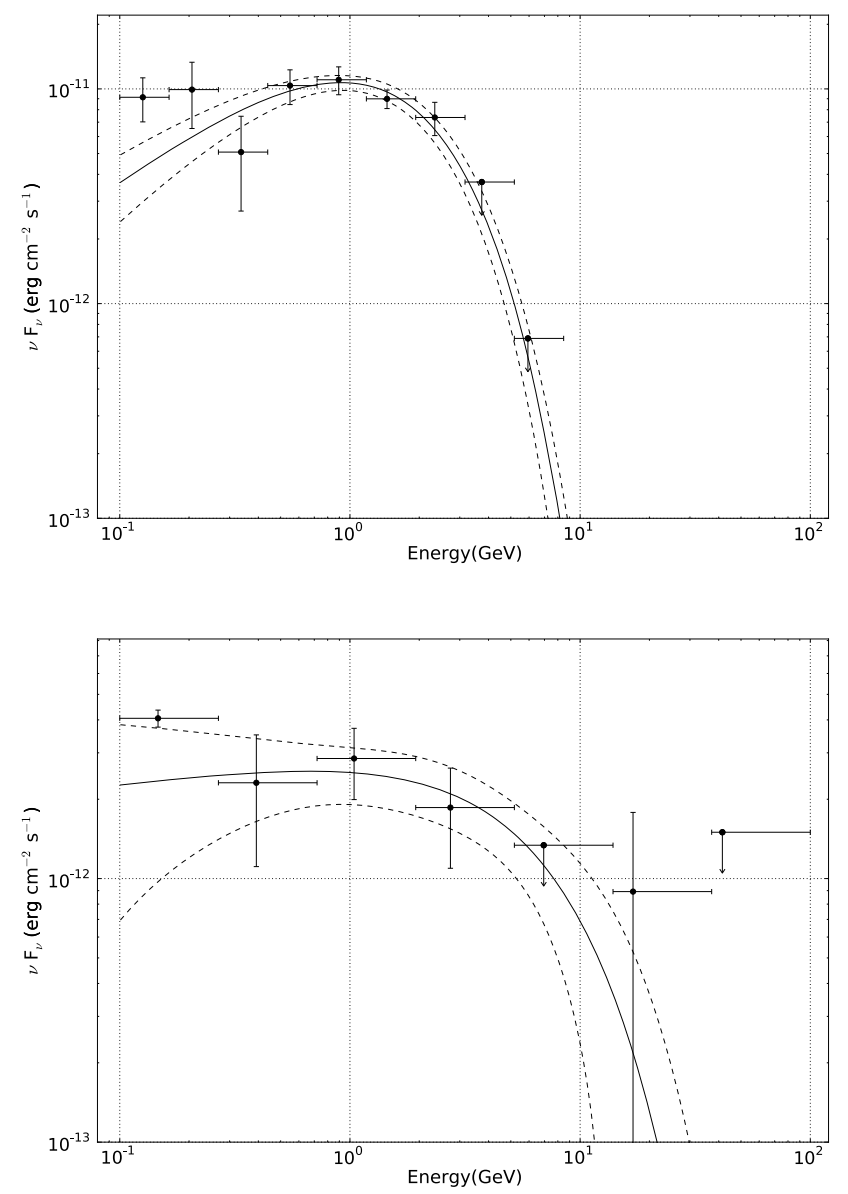

Fig. 12. On-pulse gamma-ray spectral energy distributions (SEDs) obtained with the Fermi Large Area Telescope. Top: PSR J0248+6021, in the phase range $0.2<\phi<0.55$. Bottom: PSR J2240+5832 in the phase range $0.50<\phi<0.75$. Plotted points are from power-law likelihood fits to individual energy bands with $\geq 2 \sigma$ detection significance above background for two degrees of freedom, otherwise an upper limit arrow is shown. The solid black line shows the maximum likelihood fit to a power law with exponential cutoff (Eq. (2)). The dashed lines are $\pm 1 \sigma$ uncertainties in the fit parameters.

at phase $\delta=0.39 \pm 0.02$ after the maximum of the radio peak, with a full width at half maximum $(F W H M)$ of $0.20 \pm 0.02$. The uncertainty in $\delta$ is statistical. The bias due to the $D M$ uncertainty in extrapolating the radio TOA to infinite frequency is negligible. The peak position does not vary with $\gamma$-ray energy within the statistical uncertainties, and the highest energy photon has $5 \mathrm{GeV}$ at phase 0.31 .

We fit the on-pulse $(0.2<\phi<0.55)$ data for PSR J0248+ 6021 with an exponentially cutoff power law $(\beta=1)$. The two neighbors mentioned above, as well as PSR J0218+4232 (Abdo et al. 2009a), were modeled in similar ways. Softer or sharper cutoffs $(\beta \neq 1)$ yield essentially the same statistical significance. The likelihood ratio test $(2 \Delta$ (loglike), twice the difference of the logarithm of the likelihood maximum value) prefers the simple exponentially cutoff power law $(\beta=1)$ for PSR J0248+6021 to a simple power law $(\beta=0)$ by $8 \sigma$. The spectral results for $\beta=1$ are listed in Table 1 , along with the integral photon flux $F_{100}$ and integral energy flux $G_{100}$ above $0.1 \mathrm{GeV}$. The first errors are statistical and the second are systematic. Figure 12 shows both the overall fit between 0.1 and $9 \mathrm{GeV}$ (solid lines) with $\beta=1$, 
and spectral points from likelihood fits in each individual energy band using a power-law spectrum.

We performed a maximum likelihood analysis for 50 days before and after the 2009 March glitch epoch. No change in the flux was observed. We searched the off-pulse data for a point source in the energy band $0.1-100 \mathrm{GeV}$ at the radio pulsar position. No signal was observed. After scaling to the full pulse phase, we derived a $95 \%$ confidence level upper limit on the flux of $1 \times 10^{-8} \mathrm{~cm}^{-2} \mathrm{~s}^{-1}$. If a PWN is associated with the pulsar, deep observations will be required to see it.

\subsection{PSR J2240+5832}

PSR J2240+5832 is in the Galactic plane $0.6^{\circ}$ away from the radio-quiet gamma-ray pulsar PSR J2238+5903 $\left(S_{1400}<\right.$ $7 \mu \mathrm{Jy}$, Ray et al., in prep.), and $3^{\circ}$ away from the Vela-like PSR J2229+6114 associated with the "Boomerang" PWN and SNR (Abdo et al. 2009c). No other 1FGL source lies within $3^{\circ}$. The high background from nearby PSR J2238+5903 is the reason for the choice of small value of $\theta_{68}^{\max }=0.7^{\circ}$. A total of 1208 photons with energies $>0.3 \mathrm{GeV}$ were selected. Figure 2 shows the gamma-ray pulse profiles in different energy bands, phase-aligned with the peak of the $1.4 \mathrm{GHz}$ radio profile observed at Nançay. A single peak between phases 0.55 and 0.63 appears above $0.3 \mathrm{GeV}$ only. We tried different apertures to search for a signal below $0.3 \mathrm{GeV}$, but no excess was observed at the peak location. The highest $H$-test significance is $6.3 \sigma$ and occurs above $1 \mathrm{GeV}$. Fitting above $0.3 \mathrm{GeV}$ with a Lorentzian function places the peak at $\delta=0.58 \pm 0.01$ after the radio maximum, with an FWHM of $0.11 \pm 0.02$. The peak position does not vary with $\gamma$-ray energy within statistical uncertainties, and the highest energy photon $(20 \mathrm{GeV})$ lies at $\phi=0.72$, just outside the peak phase range.

We determined the on-pulse ( $\phi=0.50-0.75)$ spectrum using the maximum likelihood analysis within a $15^{\circ}$ aperture. Both nearby pulsars PSRs J2238+5903 and J2229+6114 as well as PSR J2240+5832 were modeled by a simple exponentially cutoff power law $(\beta=1$ in Eq. (2)). Results are listed in Table 1. The source is three times fainter than the other pulsar, leading to large uncertainties in the spectral parameters. The spectral index and cutoff are consistent with those seen for most young gamma-ray pulsars. We explored different models and the exponential cutoff $(\beta=1)$ is preferred to a power law by only $2 \sigma$. Figure 12 shows both the overall fit between 0.1 and $100 \mathrm{GeV}$ (solid lines), along with the spectral points from power-law likelihood fits to each individual energy band.

As for PSR J0248+6021, we searched the off-pulse region for a possible PWN at the pulsar position. No signal was observed. Scaling to the entire phase range (a full rotation) yields a $95 \%$ confidence level upper limit on the flux of $2 \times$ $10^{-7} \mathrm{~cm}^{-2} \mathrm{~s}^{-2}$. We note however that the MILAGRO significance map for the region surrounding the "Boomerang" PWN and PSR J2229+6114 extends intriguingly towards the Galactic plane, reaching $4.7 \sigma$ at the position of PSR J2238+5903 (Abdo et al. 2009f). The Tibet air shower array similarly sees a $2.5 \sigma$ excess at that location (Amenomori et al. 2010). Both MILAGRO's and Tibet's angular resolutions are poor, and TeV PWN's are notoriously offset from the pulsars driving them. PSR J2238+5903 is more energetic $\left(\dot{E}=9 \times 10^{35} \mathrm{erg} \mathrm{s}^{-1}\right)$ than PSR J2240+5832 and presumably closer, given that it is brighter $\left(F_{100}=6.8 \pm\right.$ $1.5 \times 10^{-8}$ photons $\mathrm{cm}^{-2} \mathrm{~s}^{-1}$ above $\left.100 \mathrm{MeV}\right)$. The TeV excess might be a conflation of two adjacent sources. Deeper observations with a Cherenkov imager array may be interesting.

\section{Discussion}

\subsection{Gamma-ray luminosity}

Armed with the gamma-ray integral energy flux $G_{100}$ and the distances $d$, we can now evaluate the luminosity $L_{\gamma}$ (Eq. (1)). We set $f_{\Omega}=1$ as in Abdo et al. (2010b), and see below that the emission models in any case yield $f_{\Omega} \simeq 1$. Defining $G_{11}=$ $10^{-11} \mathrm{erg} \mathrm{cm}^{-2} \mathrm{~s}^{-1}$ and $d_{1}=1 \mathrm{kpc}=3.1 \times 10^{21} \mathrm{~cm}$ gives $L_{\gamma}=$ $1.2 \times 10^{33} f_{\Omega}\left(G_{100} / G_{11}\right)\left(d / d_{1}\right)^{2} \mathrm{erg} \mathrm{s}^{-1}$. For PSR J0248+6021, summing the distance and $G_{100}$ uncertainties in quadrature yields $L_{\gamma}=(1.4 \pm 0.3) \times 10^{34} f_{\Omega} \mathrm{erg} \mathrm{s}^{-1}$. For PSR J2240+5832, the distance ambiguity leads to larger uncertainties: if the pulsar is in the outer arm, we obtain $L_{\gamma}=(7.9 \pm 5.2) \times 10^{34} f_{\Omega} \mathrm{erg} \mathrm{s}^{-1}$, but if there is some unseen cloud along the line-of-sight creating an overdensity of electrons, and the pulsar is in the nearer Perseus arm, then $L_{\gamma}=(2.2 \pm 1.7) \times 10^{34} f_{\Omega} \mathrm{erg} \mathrm{s}^{-1}$. The corresponding efficiencies $\eta=L_{\gamma} / \dot{E}$ are listed in Table 1 .

We compare these luminosities with the rule-of-thumb for young pulsars illustrated in Fig. 6 of the Fermi Pulsar Catalog (Abdo et al. 2010b), $L_{\gamma}^{h}=10^{33} \sqrt{\dot{E} / 10^{33}} \mathrm{erg} / \mathrm{s}$, where $h$ stands for "heuristic". For the $\dot{E}$ of our two pulsars, $L_{\gamma}^{h}$ is $1.5 \times 10^{34} \mathrm{erg} \mathrm{s}^{-1}$, very near $L_{\gamma}$ obtained for PSR J0248+6021. The measured value of $L_{\gamma}$ for PSR J2240+5832 overlaps the rule-of-thumb for the distance of the Perseus arm, while the outer arm value is within the spread of values observed for the other pulsars. It is important not to assign a distance to the pulsar based on a comparison of observed $L_{\gamma}$ with expectations, since that may bias future attempts to use population modeling to refine emission models.

\subsection{Emission models}

These two pulsars have atypical gamma-ray pulse profiles: as for the six pulsars studied by Weltevrede et al. (2010), they have only one gamma-ray peak, and like four of those, they have a large offset from the radio pulse. Most gamma-ray pulsars have two peaks, the first lagging the radio beam by $<0.2$ rotations. Hence, these two pulsars help extend the parameter space over which we can test gamma-ray emission models.

To pin down the geometrical angles $\alpha$ and $\zeta$, beyond the RVM work described above, we follow Romani \& Watters (2010) in modeling the gamma-ray pulse profiles. Byproducts are the flux correction factors $f_{\Omega}$, which reduce the uncertainty in $L_{\gamma}$. We test two simple versions of outer magnetosphere pulse models. The first model ("outer gap", or OG, Romani 1996) has emission starting at the "null charge" surface and extending to the light cylinder. The second model ("two pole caustic", or TPC, Dyks \& Rudak 2003) has emission starting at the star surface and extending to a perpendicular distance of $0.75 R_{\mathrm{LC}}$ from the rotation axis or a radial distance of $R_{\mathrm{LC}}$, whichever is less. In both cases, the emission comes from a zone spanning a characteristic fraction $w=L_{\gamma}^{h} / \dot{E}=0.075$ of the open zone near the last-closed field lines. For PSR J0248+6021, we choose a linear intensity gradient across the gap but the modeled light curve shape is not very sensitive to the illumination across the gap zone, and narrow or uniformly illuminated gaps give similar results. In contrast, the very sharp $\gamma$-ray pulse of PSR J2240+5832 seems to require a relatively small range of field lines near the maximum $w$ to dominate the emission: here we used a simple Gaussian weighting, peaked around $w=0.075$. We also plot the fits to the numerical plasma-filled (force free) model of Bai \& Spitkovsky (2010). This so-called "separatrix layer" (SL) model has a dense plasma and currents and posits emission from field lines extending well outside the light cylinder into the wind zone. 
Figures 7 and 8 show the goodness-of-fit surfaces in the $(\alpha, \zeta)$ plane for the two pulsars. "Goodness-of-fit" matching of the observed and modeled post-profiles uses the exponentiallytapered $\chi_{3}$ weighting defined in Romani \& Watters (2010), a more robust test statistic than a simple $\chi^{2}$. The regions selected by the RVM fits to the radio data are superimposed. In both cases, emission from near the light cylinder (as used in this OG model) seems necessary to provide good fits. In addition, emission from below the "null charge" surface appears to create too many pulse components and too much off-pulse emission. For PSR J0248+6021, the best-fit curves in the radio-allowed region are near $\alpha=46^{\circ}, \zeta=52^{\circ}$. This model produces a broad, merged peak at the correct phase with a good fit statistic $\chi_{3}=2.4$. The inferred flux correction factor is $f_{\Omega}=1.06$. The best fit TPC model in the allowed region is near the same angles but gives a substantially poorer fit, because of a second pulse component at $\phi \sim 0.1$. Here we have $f_{\Omega}=0.86$.

A similar exercise for PSR J2240+5832 shows rather similar regions of best fit for the two models. However, again the OG model provides a better fit (near $\left.\alpha=101^{\circ}, \zeta=117^{\circ}\right)$ with $\chi_{3}=4$.1. The poorer TPC fit at this position has $\chi_{3}=7.5$. In this case, the OG light curve is dominated by a single narrow pulse near $\phi=0.6$. A much weaker component near $\phi=0.2$ is faint or missing in the present gamma-ray light curve. Although these same two components are present in the TPC curve, the first peak after the radio pulse is comparably bright to the second, and for these $\alpha, \zeta$ angles significant emission is predicted for all pulsar phases. These differences indicate that, using the particular realizations of the OG and TPC models described by Romani \& Watters (2010), the data are most consistent with the OG-type picture. For both fits, $f_{\Omega} \approx 1$. The SL models provide $\chi_{3}$ fit levels intermediate between the OG and TPC models. The relatively good fits tend to lie along a narrow band and the intersection with the RVM-allowed angles is similar to that proposed in the OG case.

Thus, while both of these pulsars show single dominant components in the $\mathrm{GeV}$ light curves, the pulse profile modeling suggests a different origin. For PSR J0248+6021, the single broad component near phase $\phi \approx 0.3-0.45$ may be a merged double from a grazing sweep across the hollow $\gamma$-ray beam. In contrast, the very narrow pulse at phase $\phi=0.6$ for PSR J2240+5832 implies a cut through a caustic surface for a component normally identified with the second peak in young pulsars: the first peak is weak or absent.

\section{Conclusions}

The Nançay survey of the northern Galactic plane led to the discovery of two remarkably similar young pulsars. They were subsequently timed for twelve years, during which period instrument precision improved steadily. The extensive timing data allowed proper motion determinations, and revealed a slowly varying $D M$ for one pulsar. These data, together with $R M$ measurements obtained with polarization data, proved crucial in constraining the distance of PSR J0248+6021 to $2 \mathrm{kpc}$, much less than the value deduced from the $D M$ alone. The timing data also facilitated investigations of the pulsar's environment. Radio polarization data constrained the pulsar geometry.

Both pulsars have large spin-down power and are among the growing number of gamma-ray pulsars detected with the Fermi Large Area Telescope. Yet they are in the minority of gamma-ray pulsars displaying only a single peak, with a large offset from the radio pulse. Gamma-ray emission modeling constrains the regions where the electrons radiate at high energies.
The gamma-ray luminosities obtained reinforce the growing evidence for how emission depends on spin-down power.

We have studied radio, optical, X-ray, and TeV data for evidence of either accompanying pulsar wind nebulae or a bright optical pulsar. None was seen, but we presented evidence to encourage deeper X-ray and TeV searches. Sustained, accurate pulsar timing combined with multi-wavelength data are thus allowing progress in the understanding of the emission mechanisms at work around neutron stars, and of the diffuse Galactic radiations at all energies.

Acknowledgements. We thank Simon Johnston for useful discussions regarding the radio polarization signal. The Nançay Radio Observatory is operated by the Paris Observatory, associated with the French Centre National de la Recherche Scientifique (CNRS). This research made use of the WEBDA database, operated at the Institute for Astronomy of the University of Vienna. The Fermi LAT Collaboration acknowledges generous ongoing support from a number of agencies and institutes that have supported both the development and the operation of the LAT as well as scientific data analysis. These include the National Aeronautics and Space Administration and the Department of Energy in the United States, the Commissariat à l'Energie Atomique and the Centre National de la Recherche Scientifique/Institut National de Physique Nucléaire et de Physique des Particules in France, the Agenzia Spaziale Italiana and the Istituto Nazionale di Fisica Nucleare in Italy, the Ministry of Education, Culture, Sports, Science and Technology (MEXT), High Energy Accelerator Research Organization (KEK) and Japan Aerospace Exploration Agency (JAXA) in Japan, and the K. A. Wallenberg Foundation, the Swedish Research Council and the Swedish National Space Board in Sweden. Additional support for science analysis during the operations phase is gratefully acknowledged from the Istituto Nazionale di Astrofisica in Italy and the Centre National d'Études Spatiales in France.

\section{References}

Abdo, A. A., Ackermann, M., Atwood, W. B., et al. 2009a, Science, 325, 848 (Millisecond Pulsars)

Abdo, A. A., Ackermann, M., Atwood, W. B., et al. 2009b, ApJ, 699, L102 (PSR J0205+6449)

Abdo, A. A., Ackermann, M., Atwood, W. B., et al. 2009c, ApJ, 706, 1331 (PSRs J1048-5832 and J2229+6114)

Abdo, A. A., Ackermann, M., Atwood, W. B., et al. 2009d, ApJ, 701, L123 (LSI+61 $\left.{ }^{\circ} 303\right)$

Abdo, A. A., Ackermann, M., Atwood, W. B., et al. 2009e, Astrop. Phys., 32, 193 (On-orbit calibration)

Abdo, A. A., Allen, B. T., Aune, T., et al. 2009f, ApJ, 700, L127

Abdo, A. A., Ackermann, M., Ajello, M., et al. 2010a, ApJS, 188, 405 (1FGL catalog)

Abdo, A. A., Ackermann, M., Ajello, M., et al. 2010b, ApJS, 187, 460

Acciari, V. A., Beilicke, M., Blaylock, G., et al. 2008, ApJ, 679, 1427

Albert, J., Aliu, E., Anderhub, H., et al. 2006, Science, 312, 1771

Amenomori, M., Bi, X. J., Chen, D., et al. 2010, ApJ, 709, L6

Atwood, W. B., Abdo, A. A., Ackermann, M., et al. 2009, ApJ, 697, 1071 (LAT)

Backer, D. C., Dexter, M. R., Zepka, A., et al. 1997, PASP, 109, 61

Bai, X.-N., \& Spitkovsky, A. 2010, ApJ, 715, 1282

Blaskiewicz, M., Cordes, J. M., \& Wasserman, I. 1991, ApJ, 370, 643

Burrows, D. N., Hill, J. E., Nousek, J. A., et al. 2005, Space Sci. Rev., 120, 165

Cognard, I., \& Theureau, G. 2006, Present and Future of Pulsar Astronomy, 26th IAU meeting, Joint Discussion 2, 16-17 August, Prague, Czech Republic, \#36, 2

Cognard, I., Theureau, G., Desvignes, G., \& Ferdman, R. 2009, in Windows on the Universe, XXI Rencontres de Blois [arXiv: 0911.1612]

Condon, J. J., Cotton, W. D., Greisen, E. W., et al. 1998, AJ, 115, 1693

Cordes, J. M., \& Lazio, T. J. W. 2002, unpublished [arXiv: astro-ph/0207156]

de Jager, O. C., \& Büsching, I. 2010, A\&A, 517, L9

Dyks, J., \& Rudak, B. 2003, ApJ, 598, 1201

Edwards, R. T., Hobbs, G. B., \& Manchester, R. N. 2006, MNRAS, 372, 1549

Foster, R. S., Ray, P. S., Lundgren, S. C., et al. 1996, in ASP Conf. Ser., 105, IAU Colloq., 160, Pulsars: Problems and Progress, ed. S. Johnston, M. A. Walker, \& M. Bailes, 25

Foster, R. S., Ray, P. S., Cadwell, B. J., et al. 1997, in BAAS, 29, 1392

Gehrels, N., Chincarini, G., Giommi, P., et al. 2004, ApJ, 611, 1005

Han, J. L., Manchester, R. N., Lyne, A. G., Qiao, G. J., \& van Straten, W. 2006, ApJ, 642, 868 
Hobbs, G. B., Lyne, A. G., Kramer, M., Martin, C. E., \& Jordan, C. 2004, MNRAS, 353, 1311

Hobbs, G., Lorimer, D. R., Lyne, A. G., \& Kramer, M. 2005, MNRAS, 360, 974

Hotan, A. W., van Straten, W., \& Manchester, R. N. 2004, Publications Astron. Soc. Aust., 21, 302

Johnston, S., Kramer, M., Karastergiou, A., et al. 2007, MNRAS, 381, 1625

Kalberla, P. M. W., Burton, W. B., Hartmann, D., et al. 2005, A\&A, 440, 775

Karastergiou, A., \& Johnston, S. 2007, MNRAS, 380, 1678

Kargaltsev, O., \& Pavlov, G. G. 2008, in 40 Years of Pulsars: Millisecond Pulsars, Magnetars and More, ed. C. Bassa, Z. Wang, A. Cumming, \& V. M. Kaspi, Am. Inst. Phys. Conf. Ser., 983, 171

Kharchenko, N. V., Piskunov, A. E., Röser, S., Schilbach, E., \& Scholz, R.-D. 2005, A\&A, 440, 403

Mattox, J. R., Bertsch, D. L., Chiang, J., et al. 1996, ApJ, 461, 396

Mitra, D., \& Ramachandran, R. 2001, A\&A, 370, 586

Mitra, D., Wielebinski, R., Kramer, M., \& Jessner, A. 2003, A\&A, 398, 993

Morgan, L. K., Urquhart, J. S., \& Thompson, M. A. 2009, MNRAS, 400, 1726

Radhakrishnan, V., \& Cooke, D. J. 1969, Astrophys. Lett., 3, 225

Ransom, S. M., Cordes, J. M., \& Eikenberry, S. S. 2003, ApJ, 589, 911

Ravi, V., Manchester, R. N., \& Hobbs, G. 2010, ApJ, 716, L85

Ray, P. S., Cadwell, B. J., Lazio, T. J. W., et al. 1999, in BAAS, 31, 903

Reich, W. 1982, A\&AS, 48, 219

Reid, M. J., Menten, K. M., Zheng, X. W., et al. 2009, ApJ, 700, 137

Romani, R. W. 1996, ApJ, 470, 469

Romani, R. W., \& Watters, K. P. 2010, ApJ, 714, 810

Roming, P. W. A., Kennedy, T. E., Mason, K. O., et al. 2005, Space Sci. Rev., 120,95

Smith, D. A., Guillemot, L., Camilo, F., et al. 2008, A\&A, 492, 923

Strong, A. W., Moskalenko, I. V., \& Reimer, O. 2004a, ApJ, 613, 962

Strong, A. W., Moskalenko, I. V., Reimer, O., Digel, S., \& Diehl, R. 2004b, A\&A, 422, L47

Theureau, G., Coudreau, N., Hallet, N., et al. 2005, A\&A, 430, 373

Tian, W. W., Leahy, D. A., \& Li, D. 2010, MNRAS, 404, L1

Watters, K. P., Romani, R. W., Weltevrede, P., \& Johnston, S. 2009, ApJ, 695, 1289

Weltevrede, P., Stappers, B. W., Rankin, J. M., \& Wright, G. A. E. 2006, ApJ, 645, L149

Weltevrede, P., Abdo, A. A., Ackermann, M., et al. 2010, ApJ, 708, 1426

Wright, E. L. 2010, in AAS Abstracts, Am. Astron. Soc. Meeting, 216, 104.01

1 Laboratoire de Physique et Chimie de l'Environnement et de l'Espace, LPC2E UMR 6115 CNRS, 45071 Orléans Cedex 02, and Station de radioastronomie de Nançay, Observatoire de Paris, CNRS/INSU, 18330 Nançay, France

e-mail: theureau@cnrs-orleans.fr
2 Space Science Division, Naval Research Laboratory, Washington, DC 20375, USA

e-mail: dparent@ssd5.nrl.navy.mil

3 George Mason University, Fairfax, VA 22030, USA

${ }^{4}$ Université Bordeaux 1, CNRS/IN2P3, Centre d'Études Nucléaires de Bordeaux, Gradignan, CENBG, Chemin du Solarium, BP 120, 33175 Gradignan, France e-mail: smith@cenbg.in2p3.fr

5 Department of Astronomy and Radio Astronomy Laboratory, University of California, Berkeley, CA 94720, USA

${ }^{6}$ Laboratoire AIM, CEA-IRFU/CNRS/Université Paris Diderot, Service d'Astrophysique, CEA Saclay, 91191 Gif-sur-Yvette, France

7 National Research Council Research Associate, National Academy of Sciences, Washington, DC 20001, USA

8 W. W. Hansen Experimental Physics Laboratory, Kavli Institute for Particle Astrophysics and Cosmology, Department of Physics and SLAC National Accelerator Laboratory, Stanford University, Stanford, CA 94305, USA

9 Center for Research and Exploration in Space Science and Technology (CRESST) and NASA Goddard Space Flight Center, Greenbelt, MD 20771, USA

10 Department of Physics and Department of Astronomy, University of Maryland, College Park, MD 20742, USA

11 High Performance Technologies, Inc., 11955 Freedom Drive, Reston, VA 20190-5673, USA

12 Max-Planck-Institut für Radioastronomie, Auf dem Hügel 69, 53121 Bonn, Germany

13 NASA Goddard Space Flight Center, Greenbelt, MD 20771, USA

14 Observatoire de Paris-CNRS/LERMA, 77 Av. Denfert Rochereau, 75014 Paris, France

15 Department of Physics and Astronomy, University of Calgary, Calgary, AB, T2N 1N4, Canada

16 National Astronomical Observatories of China, Beijing 100012, PR China 